\title{
Alle origini della prova scientifica: la scuola di polizia di Salvatore Ottolenghi
}

\author{
The Origins of Scientific Evidence: Salvatore \\ Ottolenghi's Police School
}

\author{
Loredana Garlati ${ }^{1}$ \\ Università degli Studi Milano Bicocca, Italia \\ loredana.garlati@unimib.it \\ http://orcid.org/0000-0003-2677-3100
}

\begin{abstract}
II saggio ripercorre le tappe che portarono alla nascita della Scuola di polizia scientifica (poi Scuola superiore a partire dal 1925), grazie all'opera di Salvatore Ottolenghi. La Scuola, istituita a Roma nel 1902, si proponeva di insegnare sia ai funzionari di pubblica sicurezza che a quelli della polizia giudiziaria un metodo scientifico per assolvere al meglio le proprie funzioni: nell'un caso la prevenzione dei reati, nell'altro fornire all'autorità giudiziaria dati "oggettivi" ai fini dell'accertamento della verità processuale. L'analisi è l'occasione per aprire uno squarcio su un periodo culturalmente vivace e di fideistico entusiasmo verso le cd. scienze ausiliarie (l'antropologia, la psicologia, la medicina legale, la statistica etc.), che irrompono sulla scena del processo penale, grazie anche all'impulso della Scuola positiva. Oggetto di attenzione sarà in particolare l'antropometria, messa a punto da Bertillon, e la dattiloscopia, grazie anche agli studi dell'italiano Gasti. Siamo agli albori della prova scientifica, che allora, come oggi, interroga sul ruolo del giudice, sulla legittimità dell'uso di pratiche tacciate di invasività e violazione dei diritti della persona, sul rapporto tra scienza e diritto e tra prova scientifica e discrezionalità (o libero convincimento) del giudice.

Parole chiave: Scuola di polizia scientifica; Ottolenghi, Salvatore; Antropometria; Dattiloscopia.
\end{abstract}

1 Professore ordinario di Storia del diritto medievale e moderno, Università degli Studi Milano Bicocca. 
ABSTRACT: The essay deals with the steps that led to the birth of the Scientific Police School (Higher School from 1925), thanks to the work of Salvatore Ottolenghi. The School was founded in 1902 in Rome and aimed to teach both police and investigative police officers a scientific method to best perform their tasks: in the former case, the prevention of crimes; in the latter one, providing the judiciary with "objective" data in order to ascertain the procedural truth. The survey gives the chance to focus on a culturally lively period, in which the fideistic enthusiasm towards the so-called auxiliary sciences (anthropology, psychology, forensic medicine, statistics, and so forth) came on the scene of criminal trials, also thanks to the boost given by the Positive School. Particular attention was paid to anthropometry, developed by Bertillon, and dactyloscopy, also thanks to the studies of the Italian Gasti. It was the dawn of the so-called scientific proof, which raised questions - asked even today - about the role of the judge; the legitimacy of practices accused to be invasive and to violate personal rights; the relationship between science and law and between scientific proof and discretionary power (or intime conviction) of the judge.

Keywords: Scientific Police School; scientific proof; Ottolenghi, Salvatore; Anthropometry; Dactyloscopy.

Sommarıo: Introduzione; 1. La polizia tra accusa e difesa; 2. Nuovi metodi per una polizia scientifica; 3. La Scuola di Polizia scientifica: un orgoglio nazionale; Considerazioni conclusive; Bibliografia.

\section{INTRODUZIONE}

«Quando la persona non è nota, si descriverà la sua effigie, statura et habito, dicendo, quidam homo staturae magnae, vel parvae, vel communis, habens barbam flavam, vel nigram, vel imberbis, et capillos flavos, vel nigros, longos, vel breves, oculos magnos, vel parvos, nigros, vel coesios etc. e se ha alcun segno nel volto, o nella testa, si descriva, come habens cicatricem in facie ex parte dextera, vel sinistra, vel in fronte, vel in capite, indutus vestibus longis, vel brevibus, sericeis, vel laneis, colori nigri, viridis, vel rubei etc. e in somma si descriva al meglio che si può»².

2 MASINI, Eliseo. Sacro arsenale overo Prattica dell'officio della Santa Inquisitione, Seconda Parte, Del modo di formare i processi, e essaminare Testimoni, e Rei. Bologna: Baglioni, 1665, p. 51. 
L'esigenza di descrivere imputati, arrestati o ricercati, per giungere alla loro identificazione, affonda nella notte dei tempi, ma è solo a partire dall'Ottocento che essa fu elevata a oggetto di studio, alla ricerca di una metodologia che traducesse la vaghezza espressiva, presente nel frammento del manuale di Masini, in criteri incontestabili, capaci di condurre ad esiti certi.

Per raggiungere l'obiettivo occorreva mettere a punto un sistema 'scientifico', fondato sui saperi emergenti: la medicina legale, la criminologia, l'antropologia ${ }^{3}$, le scienze sociali e psicologiche, la statistica dovevano divenire supporto imprescindibile alla conoscenza giuridica in generale, e alla penalistica in particolare ${ }^{4}$.

Questo breve saggio si propone di ripercorrere nei passaggi essenziali, e in termini inevitabilmente incompleti, la nascita della polizia scientifica in Italia. Essa va collocata nel momento in cui le indagini della magistratura iniziarono stabilmente ad avvalersi delle conquiste delle cosiddette scienze ausiliarie e di strutture di sussidio specializzate. La trasformazione, come vedremo, da empirica a scientifica riguardava tanto la polizia di pubblica sicurezza (che nel prosieguo del testo sarà indicata anche come P.S.), impiegata nei compiti di prevenzione, quanto quella giudiziaria, cui spettava fornire i tasselli del giudizio istruttorio ed eventualmente dibattimentale.

3 L'antropologia criminale, secondo la Scuola positiva, convergeva con lo scopo prefissato dal diritto penale, ossia quello di conoscere il delitto e difendersi da esso. Ai criminalisti che vi ravvisavano una minaccia per le speculazioni teoriche Ferri rispondeva che «l'antropologia criminale, anziché soppiantare il delitto penale, come da taluno si crede, non farà che porgere ad esso l'aiuto di una base positiva» [FERRI, Enrico. Diritto penale ed antropologia criminale (Studi critici sull'Uomo delinquente). Archivio di psichiatria, antropologia criminale e scienze penali, Torino e Roma, v. 1, 1880, p. 445]. Cfr. ZERBOGLIO, Adolfo. Il rinnovamento scientifico della procedura penale. La Scuola positiva, Milano, anno IV, nn. 18-19, 1894, pp. 817-831: nella prolusione al corso libero di diritto e procedura penale svolto all'Università di Pisa, l'autore dimostrava come il processo penale potesse giovarsi dell'apporto della sociologia, dell'antropologia criminale e della psicologia.

4 Il merito della scuola positiva era di aver portato nel sinedrio dei criminalisti antropologici, per lo più medici e alienisti, quel criterio distintivo giuridico che mancava per dare veste di scienza alle loro osservazioni «sbracate e dirette un po' a caso» (TURATI, Filippo. Il delitto e la questione sociale. Appunti sulla questione penale. Milano, 1883, pp. 70-71). 
La polizia, come struttura e come strumento di controllo territoriale e disciplinamento sociale e politico, è stata oggetto di studi approfonditi, soprattutto da parte degli storici delle istituzioni ${ }^{5}$, così come, negli ultimi anni, spiccano gli interessanti volumi che mettono in luce i profili tecnici dell'attività investigativa, senza tralasciare i rapporti con la scuola positiva, colmando una lacuna storiografica ${ }^{6}$.

A fronte di una così corposa letteratura, la lettura storico-giuridica che qui si propone si limita a esaminare un circoscritto campione, aprendo uno squarcio su una stagione culturale vivacissima, attraversata da profondi ripensamenti sullo statuto della scienza penale, stagione che ebbe in Salvatore Ottolenghi un indubbio protagonista ${ }^{7}$.

5 Dopo lo studio pioneristico di Achille Chiappetti (L'attività di polizia. Aspetti storici e dogmatici. Padova, 1973) si ricordano, senza alcuna pretesa di esaustività, Tra polizie e controllo del territorio: alla ricerca della discontinuità, a cura di ANTONIELLI, Livio, LEVATI, Stefano. Soveria Mannelli, 2017, in particolare TOSATTI, Giovanna. Dal «segnalamento empirico» alla polizia scientifica, pp. 403-414; TOSATTI, Giovanna. Pericolosi per la sicurezza dello Stato: le schedature della polizia tra periferia e centro. Percorsi storici, n. 0, 2011; TOSATTI, Giovanna. La repressione del dissenso politico tra l'età liberale e il fascismo. L'organizzazione della polizia. Studi Storici, Roma, n. 38, v. 1, 1997, pp. 217-255; BRUNELLO, Piero. Storie di anarchici e di spie. Polizia e politica nell'Italia liberale. Roma, 2009; SBRICCOLI, Mario. Polizia. In: SBRICCOLI, Mario. Storia del diritto penale e della giustizia. Scritti editi e inediti (19722007). t. 1. Milano: Giuffrè, 2009, pp. 373-391; MELIS, Guido. La macchina imperfetta. Immagine e realtà dello Stato fascista. Bologna, 2018.

6 Pregevoli i seguenti volumi: Salvatore Ottolenghi. Una cultura professionale per la polizia dell'Italia liberale e fascista. Antologia degli scritti (1883-1934), a cura di LABANCA, Nicola e DI GIORGIO, Michele. Milano, 2018, di cui si segnalano in particolare DI GIORGIO, Michele. Dalla medicina all'investigazione. Il contributo di Salvatore Ottolenghi per la costruzione di un sapere scientifico di polizia, pp. 37-55 e CAMPOSANO, Raffaele. La polizia scientifica in Italia, pp. 75-95, ma soprattutto pp. 81-90 e Una cultura professionale per la polizia dell'Italia liberale e fascista. Antologia del "Bollettino della Scuola di polizia scientifica (1910-1939)”, a cura di LABANCA, Nicola e DI GIORGIO, Michele. Milano, 2020.

7 Laureatosi in Medicina a Torino nel 1885, dopo una breve parentesi dedicata all'approfondimento dell'oculistica, Salvatore Ottolenghi affiancò Cesare Lombroso, suo vero maestro. Dal 1890 al 1893 fu libero docente di medicina legale nell'ateneo piemontese, che abbandonò a favore di Siena, dove ricoprì l'incarico di professore straordinario, insegnando a Medicina e a Giurisprudenza. La sede toscana non sarebbe stata l'ultima: dopo uno scontro con i vertici universitari, Ottolenghi approdò a Roma, dove ottenne la cattedra di 
Se l'età del rito inquisitorio aveva tentato di fondare la certezza della prova su un sistema pseudo-scientifico quale quello delle prove legali (una sorta di rudimentale applicazione di modelli matematici alla logica probatoria), fu la scuola positiva, come è noto, a imprimere un indirizzo scientista al processo «di cui l'assunto fondamentale consiste nello studiare la genesi naturale del delitto nel delinquente e nell'ambiente in cui vive, per adattare giuridicamente alle varie cause i diversi rimedi» ${ }^{8}$.

Il positivismo si nutriva di una crescente fiducia nei possibili utilizzi delle scienze 'naturali'. Si cominciò a proclamarne il necessario ingresso anche nelle aule dei tribunali per spostare l'attenzione dal reato al delinquente ( «la frase è vecchia, ma è bene ripeterla» ${ }^{9}$ ), esaminato nelle sue componenti fisiche e psichiche ${ }^{10}$. L'interesse era rivolto alla possibile

Medicina legale. Dall'insegnamento universitario (che passò presto in secondo piano) alla fondazione della Scuola di polizia scientifica (creatura cui si dedicherà con passione e cura costante) il passo fu breve, come vedremo. Insieme a D'Amelio, primo presidente della Corte di Cassazione, e a Sante De Sanctis, professore di Psichiatria e psicologia nell'Ateneo romano, fondò la Società Italiana di Antropologia e Psicologia criminale. Qui il 23 ottobre 1934 Giuseppe Falco, nuovo Direttore della Scuola di Polizia, celebrò il ricordo del collega, scomparso il 28 giugno di quello stesso anno (FALCO, Giuseppe. Salvatore Ottolenghi. Bollettino della Scuola superiore di polizia e dei servizi tecnici annessi, Roma, fasc. 22-23, 1935, pp. 3-16). Sulla vita e l'opera di Ottolenghi si veda SCHETTINI, Laura. Ottolenghi Salvatore. In: Dizionario Biografico degli Italiani. Roma, v. 79, 2013; MARTINI, Paolo e PIVA, Italo. Salvatore Ottolenghi e la criminologia positivista. In: Per una storia dell'insegnamento della medicina legale nell'Università di Siena, a cura di VANNOZZI, Francesca. Siena, 1993, pp. 59-69; BUZZANCA, Salvatore. La figura di Salvatore Ottolenghi. In http://ssai.interno. it/download/allegati1/instrumenta_16_14_buzzanca.pdf; LABANCA, Nicola. Salvatore Ottolenghi e i saperi di polizia. Per una biografia. In Ottolenghi Salvatore. Una cultura professionale cit., pp. 17-35. Vedi infra, § 3 .

8 FERRI, Enrico. Sociologia criminale. Napoli, 1892, p. 4.

9 «Sarà dunque il delinquente che, in seguito al delitto, verrà giudicato; non il delitto cui venga astrattamente decretata una pena» (FERRI, Enrico. Sociologia criminale cit., p. 622).

10 La contaminazione tra penale e scienze medico-sociali avvenne con cautela: «Una singolare apprensione accompagna i primi criminologi e sociologi positivisti che si avventurano sul terreno del diritto penale. Li si sente procedere con la trepidazione del pascolatore abusivo», consapevoli della «carica innovativa, anzi sovvertitrice, della prospettiva scientifica che si apprestavano a mettere in campo» (SBRICCOLI, Mario. La penalistica civile. Teorie e 
classificazione dei delinquenti e all'influenza dei fattori economico-sociali, piuttosto che alla dimensione oggettiva e 'astratta' del reato. Si intendeva investigare soprattutto il movente del fatto illecito per individuarne cause e fattori di origine e giungere così a una individualizzazione della pena ${ }^{11}$. Si osservava «una grave e profonda lacuna nella scienza del diritto penale, consistente nel difetto di una positiva conoscenza del delinquente e del delitto, ed in conseguenza nell'ignoranza dell'ufficio vero del magistero di repressione»: un vuoto colmabile solo abbandonando il metodo deduttivo per adottare quello che «tanti vantaggi ha portato alle scienze naturali» ${ }^{12}$. Erano esiti che pure gli avversari della scuola positiva, pur nella critica aspra, si vedevano costretti a riconoscere ${ }^{13}$.

Dal punto di vista processuale ciò significava una sorta di rivoluzione; «a nuovi orizzonti del diritto, nuovi orizzonti della procedura penale» ${ }^{14}$ : innanzitutto il ricorso a periti giudiziari, professionisti e specializzati nelle diverse discipline, da costituire stabilmente presso ogni ufficio di istruzione. Antropologi, psichiatri, alienisti, criminalisti avrebbero dovuto sostituire con la loro competenza il 'buon senso comune' (o, per dirla con Beccaria, «l'ignoranza che giudica per sentimento» ${ }^{15}$ )

ideologie del diritto penale nell'Italia unita. In: SBRICCOLI, Mario. Storia del diritto penale cit., t. I cit., 2009, p. 549).

11 PIFFERI, Michele. L'individualizzazione della pena. Difesa sociale e crisi della legalità penale tra Otto e Novecento. Milano, 2013.

12 PUGLIA, Ferdinando. Prolegomeni allo studio del diritto repressivo. Roma Torino Firenze, 1883, pp. 10-11.

13 Sia pure a denti stretti, Pessina ammetteva che «i progressi delle scienze naturali hanno potentemente contribuito, e sempre più contribuiranno, a rischiarare i terribili problemi della giustizia penale, oltre quelli che si rannodano al bisogno legittimo della prevenzione del delitto» (PESSINA, Enrico. Il naturalismo e le scienze giuridiche. Discorso inaugurale letto nella Regia Università di Napoli il 17 dicembre 1878. Napoli, 1879, p. 16). Brusa, critico accanito della scuola positiva, cui non risparmiava invettive feroci, era costretto a convenire, sia pure con qualche riserva, sui vantaggi derivanti dall'applicazione delle scienze al tema dell'imputabilità criminosa (BRUSA, Emilio. La morale $e$ il diritto criminale al limbo. Discorso inaugurale letto nella Regia Università di Torino l'8 marzo 1880. Torino, 1880).

14 BERENINI, Agostino. Azione ed istruzione penale. Organi e funzioni. Parma, 1888, p. VII.

15 BECCARIA, Cesare. Dei delitti e delle pene. Edizione Nazionale delle Opere di Cesare Beccaria, I, Milano, 1984, § XIV, p. 59. 
di cui i giudici si erano sempre avvalsi per penetrare nella complessa articolazione della mente umana e trarne conseguenze sul piano giuridico. Richiesta ovvia per una corrente che si proponeva piuttosto la riduzione dei delitti che delle pene e per la quale il libero arbitrio individuale cedeva di fronte al condizionamento sociale, così come all'imputabilità morale si sostituiva una responsabilità del contesto ambientale e culturale. La prevenzione e la difesa sociale, e non l'accanimento punitivo e retributivo, erano gli obiettivi perseguiti. La pena pertanto doveva prescindere da una valutazione proporzionalistica legata all'intrinseca gravità del delitto e discendere dal grado di temibilità dell'agente, considerato come l'indice più sicuro per determinare la qualità e il grado della reazione sociale alle azioni antisociali.

Ciò significava disseminare l'intera procedura di competenze scientifiche, nella speranza di «trasformare il processo in una diagnosi nosografica dell'imputato ${ }^{16}$. La metamorfosi avrebbe dovuto riguardare non solo il magistrato nella fase istruttoria, ma anche la polizia. Era infatti negli uffici di quest'ultima che bisognava cominciare ad operare la distinzione tra galantuomo e briccone. Solo facendo retroagire alla fase della cattura la tassonomia predicata da alcuni esponenti dei 'nuovi orizzonti della scienza' si sarebbe potuto evitare di giungere a sentenze di condanna o di assoluzione basandosi su informazioni spesso vaghe o indagini ispirate più dall'intuito individuale che da un metodo razionale. La creazione di una polizia scientifica si presentava per di più come l'occasione perfetta per dare attuazione alle idee della scuola positiva, princìi che invece faticavano ad essere accettati nel milieu dei giuristi, timorosi di sconvolgere capisaldi come la responsabilità individuale e il fondamento della pena ${ }^{17}$.

16 MILETTI, Marco Nicola. La follia nel processo. Alienisti e procedura penale nell'Italia postunitaria. Acta Histriae, Koper, n. 15, v. 1, 2007, p. 326.

17 «Una organizzazione razionale di un servizio di polizia, oltre ad arrecare un grande vantaggio all'ordine e alla sicurezza pubblica, avrebbe dato modo» agli studi della scuola positiva e alle «verità scientifiche» di elevarsi a sistema, in modo che il loro progresso non fosse più lasciato a ricerche individuali, «meno efficaci, troppo laboriose» (PACINOTTI, Giovanni. Una Scuola di polizia scientifica nell'Università di Ferrara. Rivista di diritto penale e sociologia criminale, Pisa, anno V, 1904, p. 311). 


\section{LA POLIZIA TRA ACCUSE E DIFESA}

La nascita della polizia scientifica ${ }^{18}$, contrapposta a quella empirica ${ }^{19}$, quale era stata concepita fino a quel momento ${ }^{20}$, rispondeva all'esigenza di utilizzare il progresso scientifico delle scienze biologiche e fisiche $^{21}$, in modo che i funzionari di Pubblica Sicurezza fossero preparati ad una lotta al delitto in una prospettiva preventiva. Fondamentale poi la formazione di una polizia giudiziaria ${ }^{22}$ chiamata non ad apprendere

18 «La polizia scientifica non è una scienza a sé ma l'applicazione di metodi razionali, scientifici nelle varie funzioni della polizia» (FALCO, Giuseppe. "Identità". Metodo scientifico di segnalamento e di identificazione. Roma, 1922, p. 27).

19 A fronte di quanti sostenevano che la polizia era empirica per natura e non necessitava né di notizie teoriche né di dati scientifici, si ribatteva che non si poteva ignorare quella messe di progressi che avrebbero potuto consentire alla polizia di condurre al meglio la battaglia quotidiana contro la criminalità (OTTOLENGHI Salvatore. Polizia empirica e polizia scientifica. Palermo, 1897, pp. 3-12 [estr. da Rivista della polizia scientifica, anno I, fasc. 2). «L'affratellamento tra gli studi scientifici e le necessità delle applicazioni pratiche è già a buon punto in parecchi campi, soprattutto per ciò che riguarda il mondo fisico, il fisiologico, l'economico. Ma in altri campi è, pur troppo! ancora un pio desiderio; ed in altri ancora ne siamo appena ai primi approcci. Tra questi, la polizia» (ROSSI, Virgilio. Polizia empirica e polizia scientifica. Il Tachiantropometro Anfosso applicato ad una centuria di criminali. Rivista di discipline carcerarie, Roma, anno XIX, 1889, p. 533).

20 «La polizia empirica si affida interamente all'intuito istintivo, all'attitudine innata di alcuni; ed all'esperienza che la lunga pratica del servizio ha conferito ai più anziani [...]. La polizia scientifica mira a corredare il giovane funzionario, fin dall'inizio della sua carriera, delle più essenziali cognizioni fornite dalla biologia, dall'antropologia, dalla psicologia e dalla medicina legale [...] per far apprendere al giovane funzionario il metodo razionale di applicazione dei dati scientifici alla pratica» (GASTI, Giovanni. Il corso di Polizia scientifica. Roma, 1903, pp. 5-6).

21 Occorreva «volgarizzare presso tutti i Funzionari il metodo di applicazione delle conoscenze scientifiche nei quotidiani mezzi di lotta contro i delinquenti» (OTTOLENGHI, Salvatore. Ai lettori. Bollettino della Scuola di polizia scientifica e del servizio di segnalamento, Roma, fasc. 1, 1910, p. 3).

22 «Riesce agevole il rilevare che tra la polizia giudiziaria e la polizia amministrativa, come sono analogie, per il genere comune cui appartengono, sono da altra parte tra esse grandi differenze per il fine che esse si propongono». La seconda cerca di «antivenire con utili espedienti l'apparizione dei fenomeni delittuosi [...], la polizia giudiziaria invece mira alla ricerca dei reati avvenuti, allo scoprimento dei colpevoli e alla preparazione degli elementi di prova che sono necessari per potere le varie magistrature dare un giudizio conforme al 
le forme giuridiche della propria attività, quanto i criteri razionali che la presiedevano, forniti dalle altre scienze impegnate a studiare il delinquente e il reato nelle diverse sfaccettature possibili.

La mancanza di metodo, il confidare nella perspicacia dei singoli per ottenere brillanti risultati non poteva più rappresentare una strada perseguibile ${ }^{23}$. Grazie all'antropometria, ai dati sfigmografici sulle variazioni del circolo sanguigno atte a rivelare le vere pulsioni ed emozioni a prescindere dal contegno esteriore ${ }^{24}$, financo all'ipnotismo ${ }^{25}$, la polizia doveva essere posta nella condizione di limitare gli inciampi in cui era incorsa fino a quel momento, spesso determinati da una carente speculazione teorico-scientifica.

Era ormai inaccettabile che la polizia fosse fatta «così come si faceva la guerra, nei tempi eroici, tutta a casaccio, ad empirismo, dove il merito individuale di alcuni pochi in astuzia ed in forza muscolare, decideva della sola vittoria» ${ }^{26}$.

In via preliminare, però, era necessario liberare il personale di polizia da pregiudizi, elevandolo al di sopra delle critiche gratuite

vero» (CARFORA, Francesco. Polizia giudiziaria. In: Digesto Italiano, Torino, v. XVIII, parte seconda, 1906-1912, p. 1048).

${ }^{23}$ GASTI Giovanni. Il corso di Polizia scientifica cit., p. 5.

24 Vedi infra.

25 FERRI, Enrico. Sociologia criminale cit., pp. 625-627. Aleggiava tuttavia un certo scetticismo sulla possibilità che l'ipnotismo potesse aiutare nello svelamento dei delitti (LOMBROSO, Cesare. L'ipnotismo applicato alla procedura penale. Archivio di psichiatria, scienze penali e antropologia criminale, Torino e Roma, v. 8, 1887, pp. 243-246; LOMBROSO, Cesare. Studi sull'ipnotismo. Roma Torino Firenze, $3^{\mathrm{a}}$ ed., 1887; ALGERI, Giovanni. Alcuni casi di ipnotismo di criminali pazzi. Archivio di psichiatria, scienze penali e antropologia criminale. Torino e Roma, v. 8, 1887, pp. 603-612). Si conveniva, invece, sulla possibilità che esso svelasse fenomeni di autosuggestione e di menzogna, nel caso di deposizione di testimoni o di imputati (LOMBROSO, Cesare-OTTOLENGHI, Salvatore. Nuovi studi sull'ipnotismo e sulla credulità. Archivio di psichiatria, scienze penali e antropologia criminale. Torino, n. 1, v. 11, 1890, pp. 545-546; OTTOLENGHI, Salvatore. La suggestione e le facoltà psichiche occulte in rapporto alla pratica legale e medico-forense. Torino, 1900).

LOMBROSO, Cesare. L'uomo delinquente in rapporto all'antropologia, alla giurisprudenza ed alla psichiatria. Torino, quinta edizione, v. 3, 1897, p. 321. L'espressione sarà poi ripresa da ROSSI, Virgilio. Polizia Empirica e Polizia Scientifica (Idee vecchie e fatti nuovi). Aquila, 1898, p. 7. 
rivolte, in nome di un sentimentalismo morboso, dall'opinione pubblica attraverso i media, o mosse dagli avvocati, con le loro arringhe impastate di «ciarlatanismo dottrinale ${ }^{27}$. Contro la polizia si sbraitava per i pretesi arbitri, per le sospette violenze praticate, evocando gli orrori dell'inquisizione, mistificando, secondo taluni, la realtà, finendo per trasformare i delinquenti in vittime e i loro persecutori in carnefici ${ }^{28}$. La polizia aveva non solo necessità di essere difesa, ma anche di vedersi riconosciuto uno spazio proprio, trascurata com'era dagli studi che la relegavano a poche improvvisate pagine di scarso interesse ${ }^{29}$.

A ciò si aggiungeva un «infiacchimento del servizio di sicurezza pubblica» imputabile all'eccessivo garantismo introdotto dal legislatore nel rito penale: «ogni garanzia di libertà pei cittadini è una limitazione all'opera dei funzionari [...]. Ogni nuova tutela tributata alla presunzione di innocenza dell'imputato restringe il campo all'efficacia dell'indizio ed al valore del sospetto» ${ }^{30}$. Era palese un rigurgito quasi nostalgico verso alcuni profili del sistema inquisitorio ${ }^{31}$. Scorie della scuola positiva

27 ALONGI, Giuseppe. Polizia e delinquenza in Italia. Roma 1887, p. 16.

${ }^{28}$ Si assisteva a una sorta di dicotomia tra la mera disquisizione accademica, pronta a riconoscere l'importanza della Polizia, e l'opinione pubblica, che mostrava un unanime sentimento di avversione (ALONGI, Giuseppe. Polizia e delinquenza in Italia cit., pp. 8-19).

29 «I criminalisti [...] ritengono che la trattazione della materia degli istituti di polizia non è della loro competenza» (PUGLIA, Ferdinando. Prolegomeni cit., 1883, p. 73).

30 GASTI, Giovanni. L'identificazione dei delinquenti e la funzione di polizia nell'attuale momento giuridico e sociale. Prolusione al corso di Segnalamento detta alla Scuola di Polizia Scientifica il 2 maggio 1910. Bollettino della Scuola di polizia scientifica e del servizio di segnalamento, Roma, fasc. 1, 1910, pp. 68-69.

31 «La presunzione di innocenza applicata al recidivo specifico o al delinquente abituale è per me un assurdo pericoloso [...]. Noi pensiamo che si dovrebbero avere due sistemi probatori, uno completamente accusatorio basato sulla più ampia presunzione di innocenza per i delinquenti occasionali e primari; uno inquisitorio basato sulla presunzione di colpa per chi ha fatto del delitto una professione, per istinto o per abitudine» (GASTI, Giovanni. L'identificazione dei delinquenti cit., p. 72). L'autore ricalcava qui il pensiero di Ferri (e di buona parte della scuola positiva), che in un sussulto di ancien régime escludeva la presunzione di innocenza quando vi fossero prove certe o la confessione o la flagranza di reato o la recidiva di un delinquente abituale, inutile intralcio 
impastavano tali riflessioni, ammettendo in via generale garanzie agli imputati (ma nella minima misura possibile) ${ }^{32} \mathrm{e}$ al tempo stesso accusando il diritto penale di un certo lassismo, con un' «allarmante tendenza alla mitezza che pare talvolta sconfinare con la debolezza e, presso i giudici non togati, anche col sentimentalismo morboso a favore dei rei. Ne conseguono pene brevi, irrazionali, utili forse ancora contro i delinquenti occasionali e primarii, ma inefficaci, sterili, deprezzate, irrise, talvolta perfino vagheggiate dal delinquente istintivo e abituale» ${ }^{33}$. La conseguenza era «l'assillante fenomeno della recidiva che prospera come le male erbe rigermoglianti più folte e vigorose dopo la falciatura» ${ }^{34}$ : vera ossessione per i positivisti che cercavano di dare risposte convincenti a un tema centrale nel contenimento della criminalità ${ }^{35}$.

Si contrapponeva inoltre l'improba fatica della polizia nel consegnare alla giustizia i delinquenti alla debolezza dei magistrati, che svuotavano le prigioni e rimettevano in libertà i detenuti grazie a cavilli ${ }^{36}$.

alla speditezza del processo e alla logica (FERRI, Enrico. Sociologia criminale cit., pp. 597-599).

32 Si guardava pertanto con diffidenza a ogni riforma del processo che limitasse le azioni di polizia a favore di agevolazioni riconosciute all'imputato, quale la possibilità di essere assistito da un difensore fin dall'inizio del procedimento: «è da augurarsi che il sistema accusatorio non sia spinto nel procedimento penale ad estremi eccessivi e che non si esageri nella diffidenza contro gli ufficiali di polizia giudiziaria che sono gli alleati più fedeli della giustizia» (GASTI, Giovanni. L'identificazione dei delinquenti cit., p. 72).

33 GASTI, Giovanni. L'identificazione dei delinquenti cit., p. 69.

34 Nello stretto connubio tra penale sostanziale e processuale, si riteneva imprudente riformare la procedura allargandone le maglie senza procedere contemporaneamente a un inasprimento delle pene per «neutralizzare gli impulsi criminosi suggestionati da una maggior speranza di impunità» (GASTI, Giovanni. L'identificazione dei delinquenti e la funzione di polizia nell'attuale momento giuridico e sociale. Prolusione al corso di Segnalamento detta alla Scuola di Polizia Scientifica il 2 maggio 1910 (continuazione). Bollettino della Scuola di polizia scientifica e del servizio di segnalamento, Roma, fasc. 2, 1911, p. 65).

35 BRUNELLI, David. Recidiva e Scuola positiva nella disciplina del Codice Rocco. Spunti di riflessione. Diritto penale XXI secolo, Padova, n. 2, 2011, pp. 333-342.

36 Per Garofalo era come se fosse in atto il tentativo di scoraggiare gli agenti di polizia dal compiere la loro missione, minacciandoli con sanzioni nel caso in cui osassero oltrepassare limiti ben precisi: «Non è uno svago, non è uno 
Ad aumentare l'opacità dell'attività di polizia erano inoltre il legame organico che essa aveva con il Ministero dell'Interno, e, a sua volta, quello del pubblico ministero con l'esecutivo di cui era, ai sensi dell'art. 129 dell'ordinamento giudiziario del 1865, rappresentante presso l'Autorità giudiziaria: una contaminazione tra poteri che non prometteva garanzia di libertà.

La 'riabilitazione' della polizia passava sì dal reclutamento di persone integerrime e capaci, ma soprattutto da un cambio di metodologia nella ricerca dei delinquenti ${ }^{37}$ : essa veniva elevata a oggetto di attenzione come raramente era capitato fino a quel momento ${ }^{38}$, nella convinzione che si stesse prospettando un'epocale chance per arginare e prevenire scientificamente la criminalità.

\section{NUOVI METODI PER UNA POLIZIA SCIENTIFICA}

Nel giro di vent'anni quella «scienza bambina, la polizia scientifica, che, come tutte le scienze che muovono i primi passi fu accolta con sorrisi di scetticismo e con qualche barzelletta ${ }^{39}$ si affermò nella pratica. La prima innovazione riguardò le tecniche di riconoscimento ${ }^{40}$ : ad essere travolto

sport la caccia ad un delinquente. Gli agenti rischiano spesso la loro vita per fare ciò che sanno essere un loro dovere» e un legislatore, più incline a tutelare i delinquenti che le forze dell'ordine, sembrava quasi disconoscere questa realtà (GAROFALO, Raffale. Il codice per i galantuomini. Roma 1902 [estr. da Nuova antologia, fasc. 16, febbraio 1902], p. 9).

37 Per garantire una maggiore sicurezza pubblica non bastava aumentare il numero di guardie o di carabinieri; occorreva invece «dare alle guardie di P.S. un prestigio che pur troppo non hanno, e forse non meritano» (LOMBROSO, Cesare. Sull'incremento del delitto in Italia e sui mezzi per arrestarlo. Torino, 1879, p. 134).

38 «La polizia è quella fra le amministrazioni dello Stato, che tutti pretendono di conoscere e di giudicare, ma che in realtà è la meno conosciuta, e la più difficile ad essere giudicata spassionatamente e con retto criterio» (LOCATELLI, Paolo. Sorveglianti e sorvegliati. Appunti di fisiologia sociali presi dal vero. Milano, 1876, p. 11).

39 D'AMELIO, Mariano. I progressi della polizia scientifica. Bollettino della Scuola superiore di polizia e dei servizi tecnici annessi, Roma, fasc. 21, 1933, p. 259. plicazione della giustizia; per quanto però la soluzione di tale quesito possa 
dalle critiche fu l'apparentemente innocuo cartellino giudiziario (che andava a confluire nel casellario giudiziario) e il modo della sua redazione ${ }^{41}$.

Ferri aveva rapsodicamente passato in rassegna i possibili «nuovi e più sicuri mezzi per la ricerca dei colpevoli», quali «le note del tatuaggio, i lineamenti della fisionomia, e del cranio, i dati sulle condizioni fisiopsicologiche [...]. le nuove ricerche sulla sensibilità riflessa, sulle reazioni vasali ne' delinquenti». Tali strumenti, ad avviso del penalista mantovano, avrebbero reso più facile e completa la serie «delle prove di identità personale e indizii della capacità a delinquere», in grado di «far desistere da vie false gli agenti di polizia giudiziaria ed i giudici istruttori od a rendere meno dubbio il responso di assoluzione e di condanna ${ }^{42}$ : suggerimenti destinati a divenire nel tempo materia di specifici approfondimenti.

Un fatto era assodato: fotografie ${ }^{43}$, cartellini giudiziari incompleti, documenti identificativi (come passaporti, licenze per il porto d'armi o per

sembrare a prima vista molto facile, spesso invece essa è difficilissima [...]. E' compito dunque di una buona amministrazione giudiziaria di adoperarsi affinchè la identità della persona possa effettuarsi su basi meno infide delle attuali» (GIANNELLI, Augusto-PARDO, Giorgio. I sistemi di segnalazione antropometrica. Osservazioni e proposte. Rivista di discipline carcerarie, parte prima, Roma, anno XXIII, n. 10, 1898, p. 453).

41 L'art. 33 del regio decreto $1^{\circ}$ dicembre 1899 n. 6509, che riformava la materia riguardante il casellario giudiziale come disciplinato dall'art. 1 del regio decreto del 6 dicembre 1865, n. 2144, prevedeva che un casellario giudiziale fosse istituito in ogni tribunale penale (oggetto poi di ulteriori interventi nel 1902 e nel 1906). Con circolare del 12 aprile 1866, il Ministero di Grazia e Giustizia aveva sollecitato il rispetto del Regolamento per la esecuzione del Reale Decreto 6 dicembre 1865. In quest'ultimo testo si definivano modi e tempi per la compilazione del cartellino (ossia l'estratto della sentenza), che doveva essere redatto entro 15 giorni dalla pronuncia di una sentenza divenuta irrevocabile (art. 10 del suddetto Regolamento), e inoltrato al Procuratore del re presso il tribunale del territorio di nascita della persona oggetto della annotazione (art. 13). Spettava poi al Procuratore annotarlo e inviarlo al cancelliere per la collocazione nel casellario, non prima che il cancelliere lo avesse a sua volta registrato in un repertorio in ordine alfabetico e posto nella "cassetta" della relativa lettera; in caso di recidiva il cartellino si aggiungeva agli altri con numerazione progressiva (artt. 14-15). Si vedano i rilievi mossi da COSENZA Vincenzo. Il casellario giudiziale. Roma, 1895, pp. 1-6. FERRI, Enrico. Sociologia criminale cit., p. 624.

43 L'identificazione mediante fotografia era nel tempo divenuta oggetto di numerose critiche: «Durant ces dix dernières annés la police parisienne a réuni plus de 100.000 photographies. Croyez-vius qu'il soit possible de comparer 
la caccia forniti dagli uffici di P.S.), compilati da cancellieri o da autorità di P.S., si presentavano carenti di dati validi e ricchi di imprecisioni ${ }^{44}$. Colore degli occhi, dei capelli, statura, forma del viso e altri tratti della fisionomia risultavano riportati in modo così generico da non risultare di alcuna utilità ${ }^{45}$. Di solito la descrizione si sintetizzava in naso, viso,

successivement chacune de ces 100.000 photographies ace chacun des 100 individus que l'on arrête quotidiennement à Paris?» (BERTILLON, Alphonse. Identification anthropométrique Instructions signalétiques. Melun, 1893, p. XIV. Ma si veda anche dello stesso autore La photographie judiciaire avec un appendice sur la classification et l'identification anthropométriques. Paris, 1890). Faceva eco per l'Italia Cosenza, il quale sottolineava l'inefficacia di una ricerca compiuta attraverso le fotografie, non solo per l'inconveniente causato dal dover esaminare migliaia di fotografie, ma per l'abilità messa in atto da chi non voleva farsi identificare. Vi è «un mezzo semplicissimo per non farsi riconoscere dagli agenti che possiedono la fotografia, ed è di alterare la forma della barba o di mutare l'acconciatura dei capelli, o di procurarsi magari qualche cicatrice sul viso». Altri accorgimenti venivano messi in atto dagli arrestati già al momento stesso della rilevazione fotografica: smorfie, alterazione della postura, espressioni insolite finivano per rendere l'immagine non corrispondente al reale (COSENZA, Vincenzo. Il casellario giudiziale cit., pp. 20-21). Lo stesso si rileva in DE NOTARISTEFANI, Raffaele. L'identificazione del delinquente (bertillonage). Napoli, 1898, pp. 11-12. Non mancavano ostacoli di carattere tecnico, quali il doversi affidare alla perizia del fotografo (non sempre all'altezza del compito). L'insieme di questi inconvenienti rendeva il ricorso alla fotografia, come metodo di riconoscimento, un debole sussidio per la polizia (ROSSI, Virgilio. Polizia Empirica cit., pp. 10-11).

Non manca chi definiva umoristiche e grottesche le informazioni così raccolte, ma soprattutto il loro uso, che impediva ai giudizi di essere «quello che devono essere e, cioè, non, come sono ora, un giuoco di sorte, ma un esame completo e sperimentabile del giudicabile» (OLIVIERI, Vittorio. Un delinquente per passione. La Scuola positiva, Milano, n. 2, 1895, p. 128). I cartellini del casellario giudiziario mostravano la loro insufficienza nel momento in cui da questura a questura si inviavano le informazioni sui connotati di pregiudicati o sospettati ricercati dalle autorità: l'approssimazione descrittiva vanificava ogni possibilità di individuazione (OTTOLENGHI, Salvatore. Il segnalamento del delinquente in servizio della polizia giudiziaria. Palermo, 1898, p. 15 (estr. da Rivista di Polizia Scientifica, n. 1, vol. 6).

Si trattava di un difetto difficile da eliminare, dal momento che ancora nel 1912, a circa dieci anni dalla fondazione della Scuola di Polizia (v. infra, § 3), Ottolenghi constatava con amarezza e disappunto come continuassero a impiegarsi espressioni quali: colorito naturale, naso arricciato o regolare, barba rasa, bocca giusta o media. «Più doloroso ancora è notare come più di una volta accade che persino da qualche Questura vengano emanate apposite circolari di ricerca di pregiudicati con un segnalamento assolutamente 
mento, bocca regolari, colorito naturale, statura media, oppure soggetto alto, magro, tozzo ${ }^{46}$, come se il tempo si fosse fermato al Sacro arsenale di Masini. Neppure soccorrevano quei segni particolari di riconoscimento che avrebbero dovuto quasi marchiare a fuoco l'identità di un soggetto: era, ad esempio, provato che una cicatrice sulla guancia era così diffusa nel mondo criminale da connotare 300 su 500 delinquenti ${ }^{47}$. Ne conseguiva che da quei documenti emergeva una sorta di 'normotipo' così anonimo da incarnarsi in "uno nessuno e centomila".

Nemmeno il nome con il quale i fuggiaschi venivano registrati era affidabile, dal momento che con facilità era possibile fornire generalità diverse da quelle reali ${ }^{48}$. Analoghe considerazioni valevano per un'inflessione dialettale o un accento, non sempre utili a ricostruire il luogo di provenienza, vista l'abilità simulatoria dei criminali. Anche altri elementi, come barba, baffi, corporatura, portamento, erano facilmente alterabili e quindi poco attendibili ai fini di provare l'identità ${ }^{49}$. Diveniva

incompleto. Quando si pensa che nell'Amministrazione della pubblica sicurezza vi sono circa 730 funzionari che frequentano la Scuola, 260 guardie di città preparate al segnalamento, che allievi ufficiali carabinieri vennero istruiti sui nuovi metodi bisogna riconoscere che l'immenso lavoro non ha ancora dato buon frutto», a dimostrazione di come l'apprendimento di tali tecniche così come il cambio di mentalità richiedessero tempo per attecchire (OTTOLENGHI, Salvatore. Il segnalamento descrittivo e le funzioni della polizia. Bollettino della Scuola di polizia scientifica e dei servizi tecnici annessi, Roma, fasc. 3, 1912, p. 79).

46 «Dove cessa la statura bassa per dar luogo alla statura media, ove è tracciato il confino tra il naso piccolo ed il medio?» (ANFOSSO, Luigi. Il casellario giudiziale centrale. Studio sulla identificazione dei delinquenti e designazione dei loro contrassegni personali. Torino, 1895, p. 7).

47 OTTTOLENGHI, Salvatore. Il segnalamento del delinquente cit., p. 15; OTTTOLENGHI, Salvatore. Il colore e la forma delle cicatrici antiche in rapporto alla medicina forense (comunicazione fatta alla R. Accademia di Medicina di Torino nella seduta del 20 dicembre 1889). Giornale della R. Accademia di Medicina di Torino, Torino, v. 37, 1889, pp. 599-617.

48 Sui modi escogitati dai recidivi (in particolare) per falsificare le proprie generalità o addirittura impossessarsi dell'identità altrui, anche attraverso una sorta di commercio dei dati cui non restavano estranee le infiltrazioni della malavita organizzata, cfr. ANFOSSO, Luigi. Il casellario giudiziale centrale cit., pp. 1-6; COSENZA, Vincenzo. Il casellario giudiziale cit., pp. 7-16.

49 «Basta avere la più breve pratica giudiziaria per sapere qual conto facciano i giudici di un indizio quasi sempre messo innanzi dagli agenti di polizia, ossia 
pertanto indispensabile mettere a punto strumenti che consentissero di procedere all'accertamento dei delinquenti in modo sicuro, perché la lotta alla criminalità e la difesa sociale passava necessariamente dalla conoscenza del "nemico" 50 . Partendo dal presupposto che la natura non crea stereotipi, ma "pezzi unici irripetibili", era necessario dotarsi delle competenze necessarie per individuare i tratti distintivi di ogni essere umano.

La colpa di tanta confusione o, peggio ancora, dell'inutilizzabilità di simili dati non poteva ricadere esclusivamente sui compilatori ${ }^{51}$, ma doveva rinvenirsi nell'idea strisciante che ci si potesse affidare alla mera percezione visiva e istintiva di ognuno, tanto fallace quanto diversamente declinabile ${ }^{52}$ : «la faccia umana si può ben dire è la pagina di un libro che non si lascia leggere che da chi ne ha appreso l'alfabeto» ${ }^{53}$.

Occorreva formare il personale di polizia per cominciare a fissare in una scheda la natura dell'arrestato, così che «la sua personalità rimarrà

la corrispondenza dei connotati» (DE NOTARISTEFANI, Raffaele. L'identificazione del delinquente cit., p. 11).

50 ANFOSSO, Luigi. La lotta contro i delinquenti. Conferenza tenuta nella società filotecnica di Torino 27 maggio 1892. Borgo San Dalmazzo, 1892, in cui si amplificava la concezione dei delinquenti come nemici interni: un esercito continuamente all'assalto, che mieteva più vittime di quanto facessero le guerre e contro il quale era necessario adottare ogni forma di difesa, in particolare di carattere preventivo, affidata proprio alla polizia.

51 Qualche responsabilità tuttavia coinvolgeva anche gli autori materiali della redazione dei cartellini, se è vero che l'80\% risultava completamente privo delle pur minime indicazioni relative ai connotati degli arrestati o ricercati (ANFOSSO, Luigi. Il casellario giudiziale centrale cit., pp. 6 e 10), mentre in altri prevaleva una certa sciatteria. Così di un tal Giuseppe l'altezza, nei cinque cartellini compilati per altrettante procedure, variava di $13 \mathrm{~cm}$.; analoga incertezza riguardava il colore dei capelli che passava dal biondo al castano scuro, mentre il naso da piccolo, via via, era divenuto grosso o il sesso era mutato, trasformandosi un tale da Mario a Maria; un ammogliato con prole era divenuto, in altro cartellino, celibe (ROSSI, Virgilio. Polizia Empirica cit., pp. 10-13).

«Nel descrivere e qualificare i connotati ha grandissima parte l'impressione soggettiva di chi li prende; un individuo che ha visto il delinquente potrà assai facilmente riconoscerlo, quando lo vede, ma se deve ad altri con mezzo di descrizioni farlo riconoscere, a meno che non esistano speciali segni caratteristici, riuscirà assai difficilmente nel suo intento» (DE NOTARISTEFANI, Raffaele. L'identificazione del delinquente cit., p.11).

OTTOLENGHI, Salvatore. Il segnalamento del delinquente cit., p. 16. 
nell'ufficio e checchè avvenga lo si potrà facilmente rintracciare» ${ }^{54}$. La sua personalità, si afferma, non solo la sua fisionomia: una precisazione rilevante, come si vedrà nel prosieguo del lavoro.

La trasformazione dell'approccio al processo di identificazione prese le mosse dal francese Alphonse Bertillon ${ }^{55}$. Questi aveva respirato fin dalla più tenera età gli originali metodi che il padre Louis Adolphe e il nonno, Achille Guillard, stavano mettendo a punto, con lo studio e la catalogazione degli individui in base ai tratti fisici ${ }^{56}$.

Alphonse si era reso conto dell'arretratezza delle conoscenze a disposizione della polizia quando, nell'ufficio di registrazione di una prefettura di polizia, si era trovato a compilare le schede che accompagnavano i singoli arrestati. L'inadeguatezza di quelle cartelle gli parve palese. Sicché, ispirandosi ora ai lavori paterni (vòlti soprattutto alla misurazione dei $\mathrm{crani}^{57}$ ) ora alle intuizioni dello statistico belga Lambert Adolphe Quetelet ${ }^{58}$, egli si convinse che occorreva sostituire la descrizione dei connotati con degli indicatori (o meglio misure) piú

54 OTTTOLENGHI, Salvatore. Il segnalamento del delinquente cit., p. 23.

55 «Alfonso Bertillon rimarrà incontestabilmente l'iniziatore della identificazione scientifica dei delinquenti che ha segnato il primo capitolo del rinnovamento completo della nostra polizia. La sua opera potrà essere perfezionata, rinnovata, ma rimarrà sempre a lui improntato il metodo scientifico delle ricerche» (OTTOLENGHI, Salvatore. Alfonso Bertillon. Bollettino della Scuola di polizia scientifica e del servizio di segnalamento, Roma, fasc. 4, 1914, p. 253).

56 BERTILLON, Suzanne. Vie d'Alphonse Bertillon. Paris 1941; LACASSAGNE, Alexandre. Alphonse Bertillon. L'homme. Le savant. La pensée philosophique. Archives de l'Anthropologie Criminelle. Lyon, t. 29, 1914, pp. 161- 166.

57 Non manca una rivendicazione italiana sulla cd. craniologia da parte di MORSELLI, Enrico. Critica e riforma del metodo in antropologia fondate sulle leggi statistiche e biologiche dei valori seriali. Roma 1880.

58 L'attenzione si rivolse in particolare verso due opere della sterminata produzione di Quetelet: Phisique sociale ou essai sur le développement des facultés de l'homme. Bruxelles, Paris, Saint-Pétersbourg, t. I, 1869 e Sur la statistique morale et les principes qui doivent en former la base. Mémoires de l'Académie Royale des Sciences, des Lettres et des Beaux-Arts de Belgique. Bruxelles, v. 21, 1848, pp. 1-68. Le teorie di Quetelet avevano suscitato ampio dibattito sullo spazio lasciato al libero arbitrio, dal momento che si sosteneva che le azioni degli uomini (comprese quelle criminali) fossero governate da leggi fisse e generali, al pari dei fenomeni della natura fisica. 
stabili, impossibili da falsare e non esposti alle oscillazioni derivanti da impressioni individuali ${ }^{59}$.

Per procedere all'identificazione degli imputati, egli unì alla capacità descrittiva i criteri dell'antropometria, ossia la misurazione delle diverse parti del corpo. L'intuizione nasceva dall'urgenza di fronteggiare una situazione che stava assumendo dimensioni allarmanti, da quando in Francia, per sfuggire all'inasprimento di sanzioni previste dalla legge 5 maggio 1885 nei confronti dei recidivi, questi ultimi avevano cominciato ad assumere con sempre più frequenza generalità diverse, senza con questo integrare gli estremi di reato ${ }^{60}$. Bertillon mise quindi a punto un metodo per l'identificazione fisica, somatica e funzionale, di una persona ${ }^{61}$.

59 BERTILLON, Alphonse. Notice sur le fonctionnement du Service d'Identification de la Préfecture de Police de Paris. Paris, 1889.

GIRI, Emilio. L'applicazione del metodo segnaletico descrittivo e le comuni prove sperimentali nella indagine giudiziaria. Bollettino della Scuola superiore di polizia e dei servizi tecnici annessi, Roma, fasc. 22-23, 1935, p. 141. In Italia la situazione non era molto diversa, dal momento che chi dichiarava false generalità incorreva in un'ammenda da cinquanta a trecento lire ai sensi dell'art. 436 del Codice penale del 1889. Non solo. L'art. 231 del Codice di procedura penale del 1865 si limitava a richiedere all'imputato di declinare le proprie generalità, senza imporre al giudice istruttore (che si guardava bene dal farlo) di accertarne la veridicità. Se per esercizio del diritto di difesa si riteneva ammissibile che l'imputato potesse mentire sulla propria colpevolezza, altrettanto non poteva dirsi per l'identità. Per Cosenza l'ipotesi integrava reato di falso, nonostante una giurisprudenza contraria che aveva finito per consentire ai delinquenti di «trarre il maggior vantaggio possibile da questa generosa aberrazione dei magistrati» (COSENZA, Vincenzo. Relazione sul casellario presentata alla Commissione per la statistica giudiziaria nella sessione del maggio 1895. Giurisprudenza italiana, Torino, v. 47, parte quarta, 1895, pp. 205-207).

Si evidenziò, tuttavia, in un impeto di orgoglio nazionalistico, che la primogenitura di tali intuizioni andava fatta risalire a Lombroso e ad Alongi. Si trattava infatti di un metodo da tempo invocato dagli antropologi italiani: cfr. LOMBROSO, Cesare. Sull'incremento del delitto in Italia cit., e ALONGI, Giuseppe. Polizia e delinquenza in Italia cit. Per Lombroso la nascita dell'antropologia criminale andava fatta risalire al 1870, quando, esaminando il teschio di un brigante, scoprì quelle anomalie che lo avrebbero condotto a elaborare il suo metodo scientifico (LOMBROSO, Cesare. Discours d'ouverture au VI Congrès d'Anthropologie criminelle. Archives d'Anthropologie criminelle, de Criminolgie et de Psychologie normale et pathologique. Lyon-Paris, t. 21, 1906, pp. 665-671). Fondamentale tuttavia restava L'uomo delinquente, risalente al 1876 , continuamente modificato fino al 1900, operazione proseguita anche 
Il sistema consisteva nel prendere un determinato numero di misure del corpo umano (undici, per la precisione), procedere alla descrizione della persona ed evidenziare gli eventuali segni particolari ${ }^{62}$. Il processo si ultimava con la fotografia del soggetto in questione (e anche su questa tecnica Bertillon non mancò di far sentire la propria voce).

L'antropometria faceva ufficialmente il proprio ingresso sulla scena dei metodi di investigazione ${ }^{63}$. Il cd. segnalamento antropometrico ${ }^{64}$ nella sola Parigi aveva consentito tra il 1883 e il 1892 di identificare circa 4.000 imputati che si videro costretti a confessare la loro vera identità (dopo aver fornito informazioni non veritiere) grazie proprio

dopo la morte di Lombroso fino al 1924. Cfr. anche BONOMI, Giuseppe. Project of an Instrument for the Identification of Persons for Use in Military Establishments, Police Offices etc. London 1872. Si trattava di uno scritto breve (dodici pagine in tutto) ma incisivo in cui veniva enunciata l'idea, poi perfezionata da Bertillon, che le misure del corpo umano fossero in grado di restituire l'unicità di ciascuno in ragione della non ripetibilità delle stesse. Se Bertillon aveva avuto il merito di avviare la polizia all'applicazione dei dati della scienza, non andava trascurato o negato che la strada era stata indicata dai nostri studiosi, come rivendicato da OTTOLENGHI, Salvatore. Il segnalamento del delinquente cit., p. 22.

${ }^{62}$ Il metodo procedeva dalla misurazione del torso (altezza, ampiezza delle braccia, misurazione del tronco) a quella della testa (ampiezza e lunghezza) e dell'orecchio destro. Seguiva poi la misurazione degli arti e la descrizione del volto (orecchio, naso, fronte, occhio), delle mani e del corpo, annotando malformazioni, segni come tatuaggi, cicatrici, deformazioni: un dettaglio minuzioso e accurato, al limite del cavilloso. Si muoveva dall'assunto che, se anche una sola di tali misure si fosse rivelata identica in due individui, sarebbe stato tuttavia quasi impossibile che fossero tutte concomitanti simultaneamente in due soggetti diversi (COSENZA, Vincenzo. Il casellario giudiziale cit., p. 22). Per una sintesi accurata cfr. DE NOTARISTEFANI, Raffaele. L'identificazione del delinquente cit., pp. 13-27; ANFOSSO, Luigi. Il casellario giudiziale cit., pp. 22-24.

63 DARMON, Pierre. Bertillon, le fondateur de la police scientifique, in L'Histoire, novembre $1987, \mathrm{n}^{\circ} 105$, pp. 43-48.

${ }^{64}$ BERTILLON, Alphonse. Sur le fonctionnement du service des signalements anthropométriques. Archives de l'anthropométrie criminel, Paris, t. 3, 1888, pp. 138- 157. Il metodo venne presentato personalmente dall'autore per la prima volta al Congresso penitenziario internazionale di Roma del 1885 e pubblicato nel Bulletin de la Société générale des prisons, Paris, t. 11, n. 3, 1887, pp. 272-297. 
alle misurazioni effettuate ${ }^{65}$. Il bertillonage (stilema coniato dal giornalista francese Pierre Brullard nel 1888 ed entrato a pieno diritto nel gergo criminologico) era applicabile essenzialmente per riconoscere eventuali recidivi $^{66}$. Per quanto complesso ${ }^{67}$, dopo un'iniziale diffidenza, venne adottato quasi ovunque ${ }^{68}$.

Secondo Bertillon, tuttavia, non ci si doveva limitare a misurare il corpo umano: come anticipato, occorreva perfezionare anche la tecnica

65 BERTILLON, Alphonse. Résultats obtenus par l'anthropométrie au point de vue de la criminalité. Rapport au Congrès international de Genève. Archives de l'anthropologie criminelle, Paris, t. 11, 1896, pp. 592-596. I dati dimostrativi della validità del metodo di Bertillon sono riprodotti in DE NOTARISTEFANI, Raffaele. L'identificazione del delinquente cit., pp. 27-28 e GIANNELLI, Augusto -PARDO, Giorgio. I sistemi di segnalazione antropometrica cit., p. 463. Si sosteneva che il successo del bertillonage fu tale da indurre i delinquenti (i quali ne erano consapevoli) ad abbandonare i paesi che lo adottavano e a trasferirsi altrove.

66 Non si trattava però soltanto di raccogliere dati attraverso queste misure, ma anche di esprimerle attraverso un preciso lessico teminologico elaborato sempre da Bertillon e via via raffinato introducendo un sistema di abbreviazione delle misurazioni effettuate. Il ricorso ad abbreviazioni rendeva più celere l'annotazione sotto dettatura. «C'est ansi, par exemple, que le mot cicatrice est représenté par les lettres cic, et le mot oblique par un simple $b$; $c$ signifie courbe, et la lettre $r$, rectiligne; $\alpha$ se lit antérieur et $\rho$ (le rho grec), posterieur; $\varepsilon$, externe, et $\mathrm{i}$ interne; $f$ se lit phalange; chaque doigt de la main est indiqué par son initiale en majuscule etc. Supponos une marque libellées ainsi: cicatrice rectiligne d'une dimension de 1 centimètre, oblique externe, sur le milieu de la deuxième phalange du médius gauche, face postérieure; elle se réduira en ligne suivante: cic. r. de l. b. $\varepsilon, m l .2$ f. M. g.. . On comprend que l'usage journalier de ces signes sténographiques donne à l'écriture une rapidité égale et même supérieure à la parole. Les agents qui s'en servent cont même jusqu'à déclarer que la lecture en est plus rapide et l'interprétation plus facile que celle de l'écriture ordinaire. Un apprentissage de quelques jour suffit pour arriver au maximum de vitesse» (BERTILLON, Alphonse. Identification anthropométrique cit., p. LXIII).

Si era tuttavia convinti che il bertillonage fosse di immediata recezione e applicazione: «basta qualche ora per apprenderl[o] e meno di una settimana per rendersi padrone di tutte le conoscenze necessarie», così da arrivare a misurare da 100 a 150 persone al giorno, ciascuna delle quali non avrebbe richiesto più di sette minuti (DE NOTARISTEFANI, Raffaele. L'identificazione del delinquente cit., p. 27).

68 Sulla diffusione del sistema antropometrico nelle diverse aree geo politiche si veda DE NOTARISTEFANI, Raffaele. L'identificazione del delinquente cit., pp. 30-44. 
fotografica, perché quelle istantanee allegate alle schede identificative sapessero contribuire a migliorare la capacità di riconoscimento degli arrestati. Si suggeriva pertanto di riprendere il sospettato, oltre che di fronte, anche di profilo ${ }^{69}$, per imprimere al meglio sulla pellicola ogni tratto del vólto, procedendo poi ad annotarlo evitando ogni approssimazione: occhi, orecchie, bocca, naso, forma del viso, guance dovevano essere descritti in modo preciso ${ }^{70}$, fino a fornire un portrait parlè $^{71}$. Solo così il casellario, costituito (lo si ricorda) dalla somma dei cartellini, avrebbe potuto rappresentare la vera e precisa biografia penale di un individuo ${ }^{72}$.

Qualche contributo provenne anche dagli studiosi italiani. A Luigi Anfosso, ad esempio, si devono l'antropometro, il craniografo e

69 Cfr. ELLERO, Umberto. La fotografia nelle funzioni di polizia e processuali. Milano 1908. A lui si deve l'invenzione delle cd. gemelle Ellero, ossia di un apparecchio fotografico costituito da due macchine poste in sincrono il modo da immortalare il criminale contemporaneamente di fronte e di profilo, come richiesto da Bertillon, ma con un unico scatto e in un'unica posa, rendendo quindi la foto segnaletica la più precisa possibile. Le gemelle vennero ben presto adottate in sostituzione dei ritrovati di Bertillon. Si veda MUZZARELLI, Federica. Formato tessera. Storia, arte, e idee in photomatic. Milano, 2003, pp. 26-27; GILARDI, Ando. Wanted! Storia, tecnica ed estetica della fotografia criminale, segnaletica e giudiziaria. Milano, 2003.

70 Il colore degli occhi, ad esempio, poteva essere codificato con 50 espressioni diverse, mentre era addirittura monumentale la descrizione delle orecchie. Per Bertillon infatti era possibile distinguere quattro 'zone' dell'orecchio (bordo, lobo, antitrago e pieghe) ciascuna delle quali suddivisibile a sua volta in quattro sottocategorie; le sedici sottocategorie erano ulteriormente scomponibili in tre o cinque 'elementi' (BERTILLON, Alphonse. Identification anthropométrique cit., pp. 66-79). A titolo di esempio, per studi particolari e curiosi, cfr. GRADENIGO, Giuseppe. L'orecchio nei delinquenti. Giornale della Regia Accademia di medicina di Torino, Torino, v. 37, 1889, pp. 223-229; GRADENIGO, Giuseppe. La conformazione del padiglione dell'orecchio nei normali, negli alienati e nei delinquenti. Giornale della Regia Accademia di medicina di Torino, Torino, v. 38, 1890, pp. 495-502; DE BLASIO, Abele. L'orecchio dei Napoletani normali e criminali. Archivio di psichiatria, neuropatologia, antropologia criminale e medicina legale, Milano-Torino-Roma, v. 26 (2 della serie 3), 1905, pp. 385-411.

71 BERTILLON, Alphonse. Identification anthropométrique cit., p. 77.

72 Così auspicava avvenisse COSENZA, Vincenzo. Il casellario giudiziale cit., p. 1. 
il tachiantropometro ${ }^{73}$, mezzi apprezzati in Italia ma poco all'estero ${ }^{74} \mathrm{e}$ concepiti per raggruppare le misure del corpo e del cranio in modo più agevole $^{75}$. Luigi Frigerio introduceva l'otometro, una sorta di compasso unito a un goniometro, utile per misurare i diametri dell'orecchio e l'angolo auricolo-temporale ${ }^{76}$. Il pletismografo, ideato da Angelo Mosso, era invece capace di «scendere nella coscienza dell'uomo più dissimulatore con esattezza matematica», senza dolore, registrando i movimenti dei vasi sanguigni: esso veniva paragonato, per la precisione dei risultati ${ }^{77}$, a

${ }^{73}$ ANFOSSO, Luigi. Il segnalamento dei delinquenti e il nuovo antropometro popolare. Archivio di Psichiatria, Scienze penale ed antropologia criminale, Torino, v. 9, fasc. 4, 1888, pp. 363-374. Se ne veda l'esaltazione in LOMBROSO, Cesare. L'uomo delinquente cit., pp. 324-27, dove l'autore rivendica con orgoglio il merito di aver dato il nome di Tachi antropometro allo strumento ideato da Anfosso, ritenuto un perfezionamento dei sistemi di Bertillon.

ROSSI, Virgilio. Polizia Empirica cit., pp. 29-51. Brusa attestava che l'uso del tachiantropometro rimase limitato alle carceri delle principali città del Regno ma senza fortuna negli altri paesi, dove il metodo Bertillon, tra entusiasmi e ravvedimenti, continuava a riscuotere maggior successo delle sperimentazioni italiane (BRUSA. Emilio, Legislazione, dottrina e giurisprudenza penale italiana negli anni 1890-91. Annuario di dottrina, legislazione e di giurisprudenza, Milano, v. 3, 1891, pp. 250-251, nt. 1).

75 ANFOSSO, Luigi. Il casellario giudiziale centrale cit., pp. 25-37 con relative illustrazioni e spiegazioni delle modalità d'uso.

76 FRIGERIO, Luigi. L'oreille externe. Étude d'anthropologie criminelle, Parys et Lion, 1888, pubblicato anche in Archives de l'anthropologie criminelle, Paris, v. 3, 1888, pp. $438-481$.

77 L'uso della pletismografia fu ad esempio centrale nel famoso caso del cocchiere Carlo Tosetti, ingiustamente accusato dell'uccisione di Veronica Zucca, una bambina di soli 5 anni. Lombroso infatti ne proclamò l'innocenza in fase istruttoria grazie anche al ricorso allo pletismografo. L'esame rivelò infatti la mancanza di reazioni vasomotorie di fronte all'immagine di crani, figure di bambine con numerose ferite o in stato di putrefazione e foto della stessa Veronica. Al contrario una reazione fu osservata alla vista di un temperino munito di lama che il Tosetti riteneva la causa del suo arresto. Inoltre, secondo Lombroso, si doveva escludere la responsabilità dell'accusato in quanto si trattava di un tipo di reato che poteva essere commesso solo da sadici o degenerati, rispondenti a precise caratteristiche psico-fisiche, assenti invece nel Tosetti. «Si vede qui, come l'antropologia criminale, se giova a scoprire il delinquente, è utile pure per riconoscere l'innocenza» (LOMBROSO, Cesare, BONELLI, Angelo. Innocenza di gravissima imputazione dimostrata dall'Antropologia criminale. Archivio di psichiatria, scienze penali ed antropologia criminale, Torino, v. 23 (v. 7 della serie 2), 1902, pp. 539-545. La citazione si trova a p. 545). Vi fu un ricorso al medesimo strumento nel caso Olivo. Pur se reo confesso di aver ucciso 
quelli ottenuti con la tortura ${ }^{78}$, e già quest'ultima equiparazione basta a intuire l'invasività del metodo pur incruento, la sua attitudine a penetrare la mente senza possibilità alcuna di opporre resistenza ${ }^{79}$.

Come ogni nuova scoperta che si basava su dati scientifici o presunti tali, l'antropometria generò un'esaltazione forse eccessiva e si assicurò seguaci ovunque.

Ma non mancarono le critiche. Si evidenziò, ad esempio, come il sistema antropometrico consentisse solo in parte di raggiungere gli scopi desiderati: seppur utile per l'identificazione di soggetti che avevano già subito una condanna, esso non impediva che quanti venivano arrestati per la prima volta potessero fornire false generalità, innescando quindi una catena di errori ${ }^{80}$.

Soprattutto la metodologia che aveva avuto ferventi adepti si rivelò ben presto farraginosa (oltre che non così infallibile come era stata contrabbandata). I cartellini assumevano dimensioni abnormi, ingestibili: la ricchezza dei dati finiva per trasformarsi in un disvalore anziché in un pregio.

la moglie, Ernestina Beccaro, e di averne smembrato il cadavere, fu assolto per mancanza di prove per ben due volte (prima dalla corte di Assise Milano, sentenza annullata dalla Cassazione, poi da quella di Bergamo). L'Olivo fu condannato a soli 12 giorni di carcere e 135 lire di multa per mero occultamento di cadavere. Lombroso fu ascoltato come perito nel secondo processo e in quella circostanza non mancò di riferire che l'Olivo, stimolato con il pletismografo, aveva reagito più al ricordo del preside del suo Istituto che alla vista della fotografia dei resti della moglie, a riprova dell'estraneità dell'uomo (LOMBROSO Cesare, BIANCHI, Augusto Guido. Il caso Olivo. Milano, 1905, figg. 8 e 10). ROSSI, Virgilio. Polizia Empirica cit., p. 16.

79 A proposito di tali misurazioni si cercò di superare l'obiezione, mossa da taluni, di una possibile violazione della riservatezza dell'arrestato, sottoposto a una serie di rilevamenti che potevano avere il vago sapore di ingerenza nella sfera personale ed intima. La preoccupazione venne aggirata osservando che, come vi era l'obbligo di declinare le proprie generalità, parimenti si era tenuti a rispondere alle altre richieste atte a giungere alla verità. Di questi interventi si sosteneva che nulla avrebbe avuto da temere l'innocente erroneamente caduto nelle maglie della giustizia: una precisazione che mirava ad impedire eventuali limitazioni normative alla libertà di azione della polizia in nome di una presunta difesa di diritti individuali.

80 COMPAGNONE, Luigi. Il casellario giudiziario ed il metodo Bertillon per accertare l'identità dei recidivi: studio e proposte. Napoli 1888. 
Così, al segnalamento antropometrico si affiancò, fin quasi a soppiantarlo, quello dattiloscopico, il quale, nato quasi in sordina (e sempre in collegamento con le scienze naturali), aveva finito per risultare maggiormente attendibile ${ }^{81}$.

$\mathrm{Fu}$ uno spiacevole episodio a determinare la crisi del sistema di Bertillon. Nel 1903, nello Stato di Washington, la polizia del penitenziario federale di Leavenworth, mentre stava procedendo all'identificazione di un prigioniero, rilevò una perfetta identità con le misure e le foto di un altro detenuto schedato tempo prima ${ }^{82}$.

Una falla si apriva dunque nella segnalazione antropometrica: la possibilità che due individui potessero presentare le stesse misure, pur se remota, non era da escludere del tutto. Solo la dattiloscopia permise, nel caso di specie, di dimostrare che i due arrestati erano soggetti diversi e ciò determinò un progressivo accreditamento delle rilevazioni delle impronte digitali. L'episodio aveva finito per mettere in discussione contemporaneamente sia il sistema di Bertillon che il ricorso alle foto segnaletiche ${ }^{83}$. L'entusiasmo mostrato verso il bertillonage fu dirottato

81 GIULIANO, Andrea. Salvatore Ottolenghi. Le impronte digitali in polizia scientifica e medicina legale. Torino, 2018.

82 LOCARD, Edmond. La preuve judiciaire par les empreintes digitales. Archives de l'anthropologie criminelle, Paris, t. 29, 1914, pp. 321-348.

83 GASTI, Giovanni. La missione attuale dell'antrometria nelle funzioni di polizia. Bollettino della Scuola di polizia scientifica e del servizio di segnalamento, Roma, fasc. 3, 1912, pp. 112-113. Un colpo mortale venne assestato in occasione del famoso furto della Gioconda al Louvre, divenuto di dominio pubblico il 23 agosto 1911 (la sottrazione del celebre quadro di Leonardo era avvenuto due giorni prima). Sulla cornice abbandonata al museo era impressa un'impronta digitale, che sembrava agevolare la ricerca del colpevole da parte della Sûreté, ma gli archivi creati con il bertillonage, per quanto all'avanguardia, non consentirono di risalire al colpevole, scoperto poi essere il lombardo Vincenzo Perrugia. Il sistema fu ufficialmente abbandonato con il congresso di Polizia scientifica di Monaco nel 1914, anche se, di fronte alla nutrita schiera di detrattori, una difesa del valore del metodo e del suo ruolo innovativo provenne proprio dagli scienziati italiani. In una comunicazione presentata al Congresso annuale dell'Associazione dei Capi di Polizia svoltosi in America nel luglio 1911, Giovanni Gasti riconosceva la «superiorità della dactiloscopia sull'antropometria per il servizio di identificazione dei delinquenti», sostenuta «fin dall'epoca in cui pochi si attentavano di affermare che potesse esservi al mondo qualche cosa di meglio del Bertillonage, imperante in tutte le polizie d'Europa e di America». Tuttavia, Gasti metteva in guardia 
verso la dattiloscopia, esaltata quasi con le stesse parole con cui si erano decantati solo pochi anni prima i risultati conseguiti con l'antropometria ${ }^{84}$ : le impronte digitali, in quanto immutabili e uniche (di nuovo il valore aggiunto era individuato nell'irripetibilità), offrivano migliori garanzie circa l'identità di un soggetto e risultavano proficuamente utilizzabili in ambito giuridico.

Fu nel 1896 che un ufficiale di polizia argentino, Juan Vucetich, propose un metodo di classificazione delle impronte digitali ${ }^{85}$. Questi sviluppò gli studi di Francis Galton (che avevano dato esiti poco soddisfacenti) ${ }^{86}$. Tali ricerche furono riprese da Giovanni Gasti, che

dalle critiche rivolte all'antropometria, «esagerate più ancora di quanto lo erano state le lodi precedenti» e dettate, a suo dire, dal desiderio di svalutare il sistema «per mettere maggiormente in evidenza la importanza del metodo delle impronte digitali, il quale non aveva certo bisogno di abbattere o di deprimere per mettersi in auge; essendo i suoi pregi così intrinseci, così assoluti, così alti, da non poter in alcun modo essere offuscati dai meriti di altri sistemi». Il sistema antropometrico restava, a detta del Gasti, un ottimo metodo di identificazione, semplicemente superato dai progressi della scienza.

84 Lo stesso Ottolenghi non esitava a collocare il segnalamento dattiloscopico «sopra tutti gli altri segnalamenti», dal momento che permetteva «la classificazione più perfetta e più ampia $[. .$.$] e la identificazione delle persone se-$ gnalate sempre e dovunque si trovino» (OTTOLENGHI, Salvatore. Relazione degli anni 1919, 1920 e 1921. Bollettino della Scuola di polizia scientifica e dei servizi tecnici annessi, Roma, fasc. 9-11, 1923, p. 19).

85 VUCETICH, Juan. Dactiloscopia comparada. El nuevo sistema argentino. La Plata, 1904. Il Vucetich aveva trovato sterile e di scarsa applicabilità pratica il metodo Galton (vedi nota successiva) e per questo lo modificò configurandone uno nuovo: l'icnofalangometria, ossia la fotografia dei polpastrelli delle dita. Se nelle prime due parti del volume l'autore evidenziava gli errori generati dal sistema antropometrico, rispetto al quale la rilevazione delle impronte digitali si prestava a un'identificazione certa, nella terza si sottolineava la superiorità del sistema dattiloscopico argentino rispetto al primitivo metodo Galton, in ragione della semplicità e della chiarezza dei 'tipi' posti a base della classificazione delle impronte stesse.

86 Francis Galton studiò le impronte digitali ritenendole un possibile ausilio per ricostruire la storia genetica di un individuo e per comprendere l'ereditarietà razziale (a lui, cugino di Charles Darwin, si deve l'eugenetica), ma seppure lo scopo prefissato non fu raggiunto, la sua classificazione delle impronte rimase una base importante di studio (GALTON, Francis. Finger prints. London, 1892), ripresa poi da Edward Richard Henry, commissario capo della polizia metropolitana di Londra (Classification and uses of finger prints. London, 1900). Proprio a Londra, una commissione, istituita nel 1893, dopo aver 
dal 1903 si dedicò a una classificazione, adottata dall'anno successivo, grazie all'approvazione dell'Ottolenghi, per l'archiviazione dei cartellini segnaletici nel casellario dattiloscopico della neonata Scuola di polizia scientifica $^{87}$. La diffusione di questa tecnica determinò un lento, quanto progressivo e inesorabile declino del sistema antropometrico ${ }^{88}$.

\section{LA SCUOLA DI POLIZIA SCIENTIFICA: UN ORGOGLIO NAZIONALE}

I metodi fin qui esposti costituirono la base per un'operazione rivoluzionaria: la creazione di una polizia scientifica in Italia ${ }^{89}$. Si trattava di intervenire su un'istituzione da sempre restia a mutamenti e innovazioni, a cui si chiedeva di affrancarsi da una mentalità ciecamente burocratica per abbracciare nuovi metodi ma soprattutto una nuova mentalità.Dopo un'iniziale cautela - al confronto con altri contesti - nell'impiego dei metodi

preso in esame il metodo Bertillon e quello Galton, propose l'applicazione riunita dei due sistemi, presentando di entrambi vantaggi e svantaggi: se il secondo si rivelava così semplice da poter essere eseguito dopo un breve tirocinio, il primo richiedeva maggiore competenza ed esperienza (DE NOTARISTEFANI, Raffaele. L'identificazione del delinquente cit., p. 30).

Sulla Scuola di polizia, v. infra, § 3.

Va rilevato che a fronte degli studi che sostenevano l'unicità e l'immutabilità delle 'figure' dei polpastrelli delle dita, i tribunali non cessarono di mostrare una certa titubanza. Si veda ad esempio la sentenza del tribunale di Pisa del 15 febbraio 1918, confermata dalla Corte d'Appello di Lucca, in cui si giunse all'assoluzione dell'imputato, chiamato in giudizio a seguito del confronto tra le impronte digitali, con la motivazione che esse non bastavano a costituire una prova convincente: se configuravano un aiuto nelle investigazioni giudiziarie, le impronte non avrebbero potuto assurgere a prova, data la intuitiva defettibilità del sistema. Il dispositivo della sentenza è riprodotto in FALCO, Giuseppe, Polizia scientifica e giurisprudenza. Bollettino della Scuola di polizia scientifica e del servizio di segnalamento, Roma, fasc. 7-8, 1921, p. 161. La diffidenza fu nel tempo superata in ragione dei risultati conseguiti, tanto che dal 1911 al 1933 si registrarono solo cinque assoluzione nonostante la prova dattiloscopica (SORRENTINO, Ugo. La prova dattiloscopica dinanzi ai magistrati italiani. Bollettino della Scuola superiore di polizia e dei servizi tecnici annessi, Roma, fasc. 22-23, 1935, p. 135).

Quando Bertillon «ideò i suoi metodi di identificazione non pensò neppure a una Polizia Scientifica anche se aveva intuito nuove applicazioni ai fini della polizia» (OTTOLENGHI, Salvatore. Una "polizia scientifica" antiscientifica. La Scuola Positiva, Milano, v. 1 dell'anno 21, 1911, p. 517). 
scientifici di identificazione dei recidivi ${ }^{90}$, esitazione che potrebbe forse ricondursi alla tradizionale predilezione del mondo giuridico italiano per un approccio formalistico o comunque teorico, era giunto per la Penisola il momento del riscatto: dalla mera importazione di tecniche elaborate altrove a una funzione di guida e di modello di riferimento per le altre nazioni, come sostenevano i fautori del nuovo approccio ${ }^{91}$. Ciò avvenne grazie soprattutto alla Scuola, sorta a Roma nel 1902 per volontà di Salvatore Ottolenghi, il quale tradusse sul campo gli insegnamenti del maestro Lombroso, rendendo realtà l'intuizione del criminologo veronese (che da tempo anelava alla formazione scientifica della polizia) ${ }^{92}$. Sebbene non vi fu un accoglimento acritico delle sue posizioni, è indubbio che la ricerca, a volte esasperata, di un collegamento tra caratteri antropologici e predittività criminale connotarono l'impostazione metodologia di Ottolenghi ${ }^{93}$, in cui si avverte una volontà classificatoria su basi bio-

OTTOLENGHI, Salvatore. Trattato di polizia scientifica. Milano, v. I, 1910, p. III.

Tuttavia l'esaltazione 'partigiana' dei fondatori della Scuola merita qualche riflessione, dal momento che la Scuola di polizia poté giovarsi sia delle scoperte messe a punto dai vari Bertillon, Galton e Vucetich (qui in sintesi ricordate), sia dei loro errori. Fu quello infatti un periodo connotato da una intensa circolazione di idee ed esperienze a livello internazionale: una condivisione di saperi che mirava non solo a una formazione 'teorica' comune (nei metodi e nel linguaggio) ma anche a una cooperazione sovranazionale nel perseguimento della criminalità.

92 Una chiara testimonianza e un'interessante chiave di lettura del rapporto tra Lombroso, Ottolenghi e l'origine della polizia scientifica è fornita dallo stesso Ottolenghi nel saggio L'opera di Cesare Lombroso e la polizia scientifica. In: L'opera di Cesare Lombroso nella scienza e nelle sue applicazioni. Milano-Torino-Roma, 1908, pp. 220-237. Dai semi gettati dal maestro sarebbe quindi sorta la Scuola di polizia scientifica, che funse da modello per altre esperienze simili. Nel 1904, ad esempio, all'Università di Ferrara si tentò di seguire l'esempio di Roma e dare vita a una Scuola di Polizia scientifica, con insegnamenti simili a quelli impartiti nella capitale (Antropologia criminale e psicologia, Polizia giudiziaria scientifica, Polizia amministrativa e di sicurezza). La Scuola si rivolgeva per lo più ai laureati in Giurisprudenza e, in modo sporadico, agli ufficiali di pubblica sicurezza, ma l'attività venne sospesa nel 1906-1907 a seguito della destinazione dei diversi direttori che si erano nel tempo succeduti a più alti incarichi (MARTINELLI, Giovani. Cenni storici su l'Università di Ferrara. Ferrara, 1908, pp. 76-77). Cfr. PACINOTTI, Giovanni. Una Scuola di polizia scientifica cit., pp. 309- 316.

93 DI GIORGIO, Michele. Dalla medicina all'investigazione cit., pp. 38-40. La classificazione dei detenuti operata da Ottolenghi riproduceva la nota 
psicologiche, al fine di creare categorie di delinquenti cui far discendere la tipologia di sanzione. La ricerca quasi maniacale della diversità morfologica di orecchie, naso, occhi, cranio o viso finivano per creare anche una sorta di gerarchia antropologia cui non era estraneo un vago sentore razziale ${ }^{94}$.

In Ottolenghi si percepisce l'eco del portato della scuola positiva, sia nell'approccio di antropologia criminale alla Lombroso sia nelle sensibilità sociologiche à la Ferri, entrambe destinate a tradursi in un certo determinismo ${ }^{95}$ : si era propensi alla criminalità per nascita, per natura, per influenza di fattori economico-sociali-familiari-ambientali la cui combinazione dava luogo a un processo estraneo e ignoto anche al soggetto che lo subiva ${ }^{96}$. Lombroso aveva invocato a suo tempo l'utilizzo da parte della polizia di tutte le conoscenze messe a disposizione dalle scienze naturali ${ }^{97}$ e non mancò Ottolenghi di riconoscere al maestro una sorta di felice illuminazione nell'occuparsi «dell'applicazione pratica nella Polizia in ispecie delle nuove scoperte scientifiche», riconducendo al breve saggio Sull'incremento del delitto in Italia e sui mezzi per arrestarlo, edito nel 1879 , l'origine della trasformazione della polizia in scientifica ${ }^{98}$.

partizione, portato della scuola positiva, tra delinquenti nati, abituali, per passione o per occasione.

94 Si rinvia all'ormai noto studio di GIBSON, Mary. Nati per il crimine. Cesare Lombroso e le origini della criminologia biologica. Milano, 2002.

95 Per Ferri il determinismo sostenuto dai positivisti si risolveva in un concetto di dipendenza intesa «nel senso di necessità naturale e non di fatalismo o predestinazione dei fenomeni umani, e quindi anche dei criminosi, dalle cause naturali» (FERRI, Enrico. Sociologia criminale. Torino, 1900, p. 350).

Da ‘lombrosiano' Ottolenghi non disdegnò di collaborare con Ferri, a sua volta fondatore della Scuola di applicazione giuridico-criminale, mentre ormai il mito di Lombroso e di una criminologia a base prevalentemente biologica stava conoscendo un certo appannamento.

97 «Dobbiamo fare nella polizia quel passo che Sadowa ci insegnò a fare nell'armata, renderla uno strumento scientifico e non routinier, che ponga in opera la fotografia, il telegrafo d'allarme, gli annunci nei giornali, e soprattutto la conoscenza dell'uomo delinquente» (LOMBROSO, Cesare. Sull'incremento del delitto cit., p. 135). broso «per primo propose ed effettuò l'applicazione di quei metodi scientifici che erano rimasti fino allora riserbati alle pure ricerche di laboratorio, negli interrogatori facendo intravvedere tutto un nuovo sistema di tortura...scientifica, che doveva dare un carattere ben più rigoroso alle inchieste giudiziarie» 
Guardando all'esperienza inglese, Lombroso invocava per la polizia italiana dei funzionari detectives e l'uso dei mezzi offerti dal progresso della civiltà ${ }^{99}$, ma in più essa avrebbe dovuto possedere gli strumenti per saper riconoscere non solo i tratti fisici ma anche psichici del delinquente. Era questo il passo ulteriore che in Italia si voleva compiere: considerare il bertillonage un capitolo di polizia scientifica, ma ampliare le indagini alle personalità del soggetto, non limitandosi alla mera identificazione fisica ${ }^{100}$. Proprio la conoscenza dell'uomo delinquente costituiva la base di una riforma che doveva prendere le mosse dai pionieristici studi lombrosiani per evitare di forgiare una polizia forse più agguerrita, ma pur sempre empirica ${ }^{101}$.

Se il bertillonage si rivelava utile soprattutto per i guardiani delle carceri $^{102}$, la polizia scientifica, concepita da Ottolenghi, doveva rivolgersi

(OTTOLENGHI, Salvatore. L'opera di Cesare Lombroso cit., p. 227). Il laboratorio diveniva un campo ben più vasto rispetto ad angusti locali di studio: era la criminalità, o sarebbe forse più corretto dire erano i delinquenti le cavie su cui sperimentare i ritrovati della biologia e della psichiatria per garantire una maggior sicurezza sociale. Sul legame tra Lombroso, Ottolenghi e la polizia scientifica si veda da ultimo, D'ANTONIO, Emanuele. Lombroso, Ottolenghi e le origini della polizia scientifica italiana. In: Una cultura professionale cit., pp. 23-44.

«Abbiamo dei questori che sono abili, come lo erano Ulisse ed Achille; ma non ne abbiamo nessuno, però, che si serva per le sue indagini delle risorse scientifiche offerte dagli studi di statistica, di antropologia criminale, che moltiplichi, insomma il proprio ingegno, colle forze enormi, e quel che è più esattamente governabili, dalla scienza» (LOMBROSO, Cesare. L'uomo delinquente cit., p. 321).

${ }^{100}$ La scuola mirava ad assumere una connotazione tutta 'italiana', interpretando il 'segnalamento' «non come fine a se stesso per l'accertamento dell'identità, ma come introduzione necessaria alla conoscenza della personalità del delinquente e in special modo della individuale pericolosità e correggibilità» (OTTOLENGHI, Salvatore. Prefazione a FALCO Giuseppe. "Identità". Metodo scientifico di segnalamento e di identificazione. Roma, 1922). Cfr. anche GASTI, Giovanni. Il corso di Polizia scientifica cit., pp. 6-8).

${ }^{101}$ Così OTTOLENGHI, Salvatore. L'opera di Cesare Lombroso cit., p. 223.

${ }^{102}$ Proprio riferendosi alle guardie carcerarie «surveillant de prison qui passe son existence en tête à tête avec des êtres humains et qui, à proprement parle, est un gardien d'hommes», Bertillon scriveva che esse «doint tendre à devenir anthropologiste» (BERTILLON, Alphonse. Identification anthropométrique cit., p. III). 
a tutti i funzionari di polizia dello Stato (sia di pubblica sicurezza che giudiziaria) e pervadere tutti gli uffici dell'amministrazione di P.S., fino all'arma dei Carabinieri, perché, qualunque fosse l'ufficio cui erano preposti, i funzionari potessero avvalersi delle scienze nell'esecuzione dei loro molteplici servizi ${ }^{103}$. Prendeva corpo l'idea di un'indagine giudiziaria non più basata sull'empirismo, ma sullo studio antro-psicologico dell'individuo ${ }^{104}$ e del suo ambiente ${ }^{105}$ : la personalità del soggetto e l'individuazione delle diverse specie dell'uomo delinquente dovevano essere al centro degli obiettivi perseguiti ${ }^{106}$.

Gli studi compiuti da Lombroso furono invocati per marcare la differenza tra gli orientamenti nostrani e quelli d'Oltr'Alpe ${ }^{107}$. All'insigne padre della criminologia andava riconosciuto il merito di aver reso possibile la conoscenza pratica del delinquente non ricorrendo alla mera osservazione intuitiva, riservata a pochi, ma mediante ricerche metodiche e sperimentalmente ripetibili. Non si trattava di basarsi sulle

103 «La conoscenza antropopsicologica del delinquente si impone al funzionario di P.S., perché egli possa coll'esempio e colla parola inculcare agli agenti quei principii che devono trasformare radicalmente i mezzi di lotta contro i prodotti della degenerazione, dell'intossicazione, del proletariato, onde assumano quella forma che il progresso della scienza e dello spirito filantropico impongono, e servano anzichè ad aizzare ad ammansire le più feroci belve umane, i così detti bruti del codice penale» (OTTOLENGHI, Salvatore. Trattato di polizia scientifica (1910) cit., pp. IX-X).

${ }^{104}$ L'antropopsicologia avrebbe consentito tanto al funzionario quanto al magistrato di interpretare la psiche dell'imputato, «per saper procedere con metodi meno infidi negli interrogatori, per poter valutare giustamente la temibilità, la capacità a delinquere» (OTTOLENGHI, Salvatore. Trattato di polizia scientifica (1910) cit., p. IX).

${ }^{105}$ OTTOLENGHI, Salvatore. Seconda conferenza internazionale di Polizia New York 12-16 maggio 1925. Bollettino della Scuola superiore di polizia e dei servizi tecnici annessi, Roma, fasc. 14-15, 1926, pp. 182-184.

${ }^{106}$ Lo scopo della Scuola di Polizia era quello di «diffondere in tutte le funzioni della polizia il metodo scientifico di indagini e di porre come base di ogni funzione di prevenzione e repressione la profonda conoscenza dell'uomo normale e dell'uomo delinquente in specie» (OTTOLENGHI, Salvatore. Polizia scientifica biologia e antropologia criminale. Bollettino della Scuola di polizia scientifica e del servizio di segnalamento, Roma, fasc. 3, 1912, p. 74).

107 DI TULLIO, Benigno. La profilassi criminale nella polizia. Bollettino della Scuola superiore di Polizia e dei servizi tecnici annessi, Roma, fasc. 14-16, 1937, pp. 88-101. 
sole misurazioni proposte da Bertillon (metodo che non fu mai rinnegato perché a suo modo contribuiva alla conoscenza somatica costituzionale del soggetto), ma di avvalersi di un approccio filogenetico nello studio del delinquente, indagando sulla sua 'discendenza', sulla adattabilità al contesto sociale, sulla sua psiche.

L'istituzione della Scuola di Polizia scientifica prendeva le mosse da lontano, dal laboratorio medico-legale istituito da Ottolenghi nell'università di Siena ${ }^{108}$ (dove deteneva la cattedra di Medicina legale quale professore straordinario e che abbandonò per la mancata promozione a professore ordinario) e dai corsi di formazione promossi in quell'Ateneo già nel $1895^{109}$. Nel 1902 Ottolenghi si trasferì a Roma ${ }^{110}$ e qui poté realizzare il suo più ambizionso disegno: fondare una Scuola per la Pubblica Sicurezza. A tale scuola, come anticipato, egli conferí un indirizzo antropo-biologico ${ }^{111}$; vi collegò, l'anno successivo, la Rivista di Polizia Scientifica, fondata con Giuseppe Alongi, questore a Palermo, e nel 1910 pubblicò il primo numero

${ }^{108}$ FRANCHI, Bruno, Il principio individualizzatore nell'istruttoria penale. La Scuola Positiva, Milano, n. 11, v. 10, 1900, p. 658.

109 OTTOLENGHI, Salvatore. L'insegnamento universitario della polizia giudiziaria scientifica. Torino, 1897, passim. Sul punto si veda BASSI, Gabriele. Salvatore Ottolenghi a Siena. In: Salvatore Ottolenghi. Una cultura professionale cit., pp. 57-74.

${ }^{110}$ Dalla cattedra romana di Medicina legale Ottolenghi poteva unire all'impegno universitario (sempre meno di suo interesse) la creazione della scuola. All'inizio forse gli esiti non furono quelli sperati, se dobbiamo dar credito a Giuseppe Falco, il quale, in occasione della commemorazione dell'amico scomparso, ricordava che il primo corso «fu frequentato da un certo numero di funzionari già in carriera: di questi la gran parte ne uscì indifferente e diffidente, ma qualcuno ne uscì convinto ed entusiasta», a dimostrazione della difficoltà di superare il misoneismo che circondava sia il fondatore sia la Scuola stessa (FALCO, Giuseppe. Salvatore Ottolenghi cit., p. 5).

${ }^{111}$ La Scuola non si proponeva solo di formare, con lezioni teoriche e attività pratiche, i funzionari della P.S., ma anche di indirizzare le decisioni politiche. Essa rivendicava infatti d'aver sollecitato Vincenzo Quaranta (reggente, per il breve arco temporale di undici mesi, della Direzione generale della pubblica Sicurezza sotto l'egida del Ministero dell'Interno guidato da Francesco Saverio Nitti) ad emanare una circolare datata 23 marzo 1920 con la quale si imponeva il segnalamento sistematico dei "giudicandi" per i reati più gravi (OTTOLENGHI, Salvatore. Relazione degli anni 1919, 1920 e 1921 cit., pp. 20-21). 
del Bollettino della Scuola di polizia scientifica ${ }^{112}$ che ebbe il merito, al pari della rivista (vissuta, però, per appena sei numeri), di divulgare i nuovi metodi dell'antropologia, della psicologia, della sociologia, della medicina legale, delle scienze fisiche e naturali113.

I corsi, per volere del Ministro Zanardelli, divennero obbligatori per tutti i funzionari di pubblica sicurezza tirocinanti a partire dal $1903^{114}$ : costoro avrebbero appreso il metodo pratico per le osservazioni

${ }^{112}$ Nel Bollettino si dava volta per volta conto del numero dei partecipanti ai diversi indirizzi in cui la scuola era articolata, dei corsi e dei programmi, dei risultati raggiunti dagli allievi, delle conferenze svolte nei periodi di riferimento. Si riportavano i principali casi esaminati, i dati relativi ai cartellini segnaletici giunti, il numero di interventi compiuti, delle indagini svolte e dei successi, da valutare «non solamente dal numero di casi in cui si venne alla identificazione del colpevole, ma ben più dal numero rilevante dei casi in cui si procedette a rilievi» (OTTOLENGHI, Salvatore. Diffusione e risultati del Servizio d'investigazione giudiziaria nel quadriennio 1915-1918. Bollettino della Scuola di polizia scientifica e del servizio di segnalamento, Roma, fasc. 7-8, 1921, p. 40). Si veda per maggiori dettagli DI GIORGIO, Michele. Un laboratorio per la costruzione e la diffusione dei saperi. La scuola di polizia scientifica e il "bollettino" (1910-1939). In: Una cultura professionale cit., pp. 47-92.

113 «La Scuola di Polizia Scientifica ha apportato, nei metodi di lotta contro il delitto, una vera e profonda trasformazione [...]. Non solo reprimere, ma più ancora poter prevenire i reati: ecco, dunque, il compito della Scuola. Compito buono e nobile che va "segnalato" alla pubblica opinione e sinceramente elogiato» (BISCEGLIA, Roberto. La Scuola di Polizia Scientifica. Noi e il mondo. Rivista mensile de La Tribuna, anno 13, n. 1, 1923, p. 838).

${ }^{114}$ Il corso venne all'inizio svolto in un piccolo locale nelle carceri di via Giulia a Roma (precisamente nella sala dei riconoscimenti di Regina Coeli, grazie anche all'interessamento di Francesco Leonardi, senatore nonché Direttore generale della P.S., e all'autorizzazione di Giolitti), messo a disposizione dal Direttore generale delle carceri Antonio Doria. Esso comprendeva tre insegnamenti: Antropologia e psicologia applicata, Investigazioni giudiziarie, Segnalamento e fotografia giudiziaria; vi si aggiunsero due corsi complementari di Polizia amministrativa e di Diritto e procedura penale applicati. Fu proprio Ottolenghi a chiedere che la Scuola sorgesse in un carcere perché «lo studio del delinquente costituiva la base essenziale della Scuola» (FALCO, Giuseppe. Salvatore Ottolenghi cit., p. 6). Dal 1904 la Scuola assunse il Servizio di segnalamento dei pregiudicati della Questura di Roma, primo passo per l'istituzione di uffici di segnalamento e fotografia giudiziaria nelle principali questure: il compito che essa si prefiggeva era di inviare i cartellini segnalatori proprio alla Scuola romana, dove, in questo modo, si formò il casellario centrale d'identità. Nel 1907 la Scuola, di nuovo per intervento di Giolitti, fu trasferita nel vicolo delle Mantellate, in un locale apposito. Nel 1909 la 
psicologiche e per interpretare le reazioni, la mimica facciale, i movimenti incoscienti, utili nel caso di interrogatori tanto di un teste, quanto di un imputato o di un pregiudicato; la polizia giudiziaria veniva invece posta nella condizione migliore per collaborare efficacemente con il magistrato.

Nel giro di circa trent'anni la Scuola si dotò di un servizio di segnalamento (di pregiudicati e imputati, a richiesta della questura di Roma e dell'autorità giudiziaria ${ }^{15}$ ) e di uno antropologico-biografico, diretto a far conoscere, con obiettivi criteri scientifici, il delinquente e la sua pericolosità. Nel 1925, dopo circa vent'anni di elaborazione, fu messa a punto anche la cosiddetta "cartella biografica del pregiudicato" ${ }^{116}$. Era

frequenza divenne obbligatoria nel «primo periodo dell'alunnato, stabilendo la durata non inferiore a tre mesi, rendendo obbligatorio l'esame finale e stabilendo che l'approvazione all'esame è indispensabile condizione per la nomina a funzionario effettivo» (OTTOLENGHI, Salvatore. La Scuola di Polizia scientifica e il servizio di segnalamento in Italia 1902-1910. Cenni storici. Bollettino della Scuola di polizia scientifica e del servizio di segnalamento, Roma, fasc. 1, 1910, pp. 5-6). Si trattava di un'evidente contraddizione tra l'obbligatorietà imposta e la mancata istituzione ufficiale, che avvenne solo con regio decreto 7 dicembre 1919 n. 2504, quando alla Scuola fu riconosciuto un regolare e stabile assetto giuridico, con la previsione al proprio interno di tre servizi tecnici: servizio centrale di segnalamento e identificazione con la formazione di un casellario centrale di identità; servizio di investigazioni tecniche di polizia giudiziaria; servizio antropologico-biografico per i delinquenti pregiudicati e sospetti. Nel 1925 la Scuola assunse il nome di Scuola superiore di Polizia, una denominazione che evidenziava il suo importante ruolo didattico (FALCO, Giuseppe. "Identità" cit., pp. 3-5. Il decreto istitutivo e il relativo regolamento si trovano riprodotti in Bollettino della Scuola di polizia scientifica e del servizio di segnalamento, Roma, fasc. 7-8, 1921, pp. 204-211). Sul punto SCHETTINI, Laura. Per lo studio della personalità del delinquente. La polizia scientifica e il servizio di identità. In: Una cultura professionale cit., pp. 93-107.

${ }^{115}$ FALCO Giuseppe. "Identità" cit., p. 6. Per l'importanza, in generale, di questo servizio cfr. OTTOLENGHI, Salvatore. Il segnalamento del delinquente cit., pp. 3-25.

116 OTTOLENGHI, Salvatore. La nuova "cartella biografica dei pregiudicati". Bollettino della Scuola di polizia scientifica e del servizio di segnalamento, Roma fasc. 5-6, 1916, p. 159; FALCO, Giuseppe. Evoluzione della "cartella biografica" e pericolosità. Bollettino della Scuola superiore di polizia e del servizio di segnalamento, Roma, fasc. 14-15, 1926, pp. 126-151; OTTOLENGHI, Salvatore. La personalità del delinquente e la "cartella italiana biografica del pregiudicato" alla IV riunione della commissione internazionale di polizia criminale (6-8 luglio 1927) ad Amsterdam. Bollettino della Scuola superiore di polizia e 
la più decisa azione dell'«orientazione delle indagini ai caratteri delle personalità; riforma tutta italiana che gli stranieri non hanno ancora compresa ${ }^{117}$. La cartella non avrebbe dovuto costituire un arido elenco di reati, ma mirava a riassumere tutto ciò che poteva servire per conoscere il sospettato, dalla sua condizione di vita, alle sue degenerazioni ereditarie o somatiche, oltre a contenere un'esposizione sistematica e razionale dei suoi precedenti.

Oltre alla cartella biografica, l'altra novità fu rappresentata dall'ideazione, da parte di Ottolenghi, del «ritratto parlante del sopraluogo» $[\mathrm{sic}]^{118}$ (sulla falsariga del "porta ritratto parlante" ideato da Bertillon), applicato per la prima volta nella Scuola di Polizia Scientifica nel $1917^{119}$. La stessa accortezza che la polizia di Pubblica Sicurezza doveva riservare alla descrizione degli arrestati andava impiegata dalla polizia giudiziaria per descrivere il luogo del reato, sempre secondo rigorosi criteri scientifici ${ }^{120}$.

del servizio di segnalamento, Roma, fasc. 16-17, 1928, pp. 131-133. Il modulo si componeva di quattro parti, in cui si riassumeva la carriera criminale del soggetto, gli avvenimenti più importanti della sua vita (a cominciare dall'ereditarietà e dal contegno tenuto nelle diverse circostanze dell'esistenza) e i caratteri psichici e fisici più spiccati per consentire al funzionario di acquisire cronologicamente e oggettivamente i dati sufficienti per un giudizio sulla specialità criminosa e la pericolosità dell'iscritto. La soddisfazione maggiore fu il riconoscimento a livello internazionale di tale strumento, adottato o imitato ovunque.

117 RUSTICUCCI, Luigi. Nel regno dei ladri. Come si ruba e come si falsificano le monete. Bologna, 1919, p. 140.

118 OTTOLENGHI, Salvatore. Relazione degli anni 1919, 1920 e 1921 cit., p. 37; GIRI, Emilio. "Il ritratto parlato del sopraluogo". La Giustizia penale (parte prima: I presupposti del diritto e della procedura penale), Roma, v. 40 (10 della $4^{\text {a }}$ serie), 1934, pp. 205-210; GIRI, Emilio. Evoluzione del ritratto parlato "Ottolenghi" del sopralluogo. Bollettino della Scuola di polizia scientifica e dei servizi tecnici annessi, Roma, fasc. 12-13, 1925, pp. 188-196; GIRI, Emilio. Il "ritratto parlato" del sopralluogo e l'art. 233 del codice di procedura penale. Bollettino della Scuola superiore di polizia e dei servizi tecnici annessi, Roma, fasc. 21, 1933, pp. 168-176; GIRI, Emilio. Il "ritratto parlato" del sopralluogo nella pratica. Bollettino della Scuola superiore di polizia e dei servizi tecnici annessi, Roma, fasc. 21, 1933, pp. 177-210.

${ }^{119}$ OTTOLENGHI, Salvatore. Trattato di polizia scientifica. Milano, v. II, 1932, p. 447.

${ }^{120}$ OTTOLENGHI, Salvatore. Diffusione e risultati del Servizio d'investigazione giudiziaria nel quadriennio 1915-1918. Bollettino della Scuola di polizia 
I rilievi descrittivi permettevano a chi ne prendeva visione di formarsi un'idea precisa sullo stato degli ambienti, sulla disposizione dei mobili e degli arredi nonché degli eventuali cadaveri rinvenuti ${ }^{121}$ (ossia del contenente e del contenuto), grazie ad una capacità di osservazione analitica. Il ritratto parlato del sopralluogo doveva costituire il documento più importante del procedimento istruttorio, capace di superare la staticità di un'immagine fotografica e utile ai fini di comprendere sia la dinamica del delitto che la personalità del delinquente ${ }^{122}$.

Non meno importante fu il contributo per la messa a punto di un metodo grafonomico, che consentiva l'applicazione dei criteri del segnalamento descrittivo-antropometrico alle indagini sulla grafia, un metodo che si affermò definitivamente come ulteriore elemento di accertamento della verità dopo il celeberrimo caso Dreyfus ${ }^{123}$.

scientifica e del servizio di segnalamento, Roma, fasc. 7-8, 1921, pp. 37-47; OTTOLENGHI, Salvatore. Introduzione alle indagini tecniche di polizia giudiziaria. Bollettino della Scuola di polizia scientifica e dei servizi tecnici annessi, Roma, fasc. 21, 1933, pp. 165-167.

${ }^{121}$ Non ci si poteva esimere dal possedere nozioni base di medicina legale «sui fenomeni cadaverici» per comprendere l'importanza di certi reperti (posizione del cadavere, macchie di sangue) e accertare così le cause della morte o sciogliere i dubbi nei casi di omicidio-suicidio (OTTOLENGHI, Salvatore. Insegnamento di investigazioni giudiziarie. Bollettino della Scuola di polizia scientifica e del servizio di segnalamento, Roma, fasc. 1, 1910, p. 9).

${ }^{122}$ Per una ricostruzione dettagliata si rinvia a DI GIORGIO, Michele. Un laboratorio cit., pp. 75-80.

${ }^{123} \mathrm{Fu}$ proprio grazie a un'attenta perizia calligrafica che venne risolto il caso. Secondo Ottolenghi sarebbe stato possibile evitare uno dei più gravi errori giudiziari della storia se l'esame del manoscritto attribuito a Dreyfus (il quale, sulla base di esso, era stato accusato di spionaggio, dal momento che la lettera anonima indirizzata all'attaché militare tedesco Maximilian von Schwartzkoppe rivelava, a giudizio degli accusatori, informazioni segrete relative alla strumentazione bellica francese) fosse stato accompagnato dallo studio delle personalità dei soggetti coinvolti, per comprenderne la capacità a delinquere (OTTOLENGHI, Salvatore. La perizia di scrittura e la identificazione grafica. Bollettino della Scuola di polizia scientifica e dei servizi tecnici annessi, Roma, fasc. 9-11, 1923, p. 142). La curiosità risiede nel fatto che fu proprio il 'mitico' Bertillon a ingannarsi sulla paternità del bordereau e a determinare la condanna di Dreyfus. Ci si era rivolti a Bertillon in ragione della fama sua personale e del suo laboratorio. Forse anche per effetto di pressioni politiche, dopo affermazioni caute, egli non aveva tardato a sostenere che il documento incriminato fosse un'autofalsificazione, tesi abilmente ribadita durante le 
Gli sforzi per formare una polizia scientifica ottennero una sorta di riconoscimento ufficiale con il Codice di procedura penale (d'ora in poi c.p.p.) del 1913, art. 166, dove si dava facoltà, anche agli ufficiali di polizia giudiziaria, oltre che ai periti, di poter eseguire i rilievi descrittivi, elevati in tal modo a metodo ordinario di indagine ${ }^{124}$. Tale disposizione non aveva precedenti nel codice del 1865, anche se la relativa "giovinezza" dei criteri adottati, in auge da circa un decennio, aveva reso il legislatore del 1913 timido e guardingo: quel possono procedere, se del caso, anche a rilievi tecnici e fotografici, locuzione contenuta nel capoverso dell'articolo, era la simbolica rappresentazione di un'evidente cautela. Si trattava comunque di un sicuro passo in avanti, cui non era estranea l'opera di persuasione di Ottolenghi e dei suoi collaboratori, che seguivano attentamente l'evoluzione legislativa in atto, sia in ambito processuale che penale ${ }^{125}$. Il confronto tra

udienze del primo processo. Il capitano Dreyfus fu descritto come un accorto contraffattore, capace di alterare volontariamente la grafia per poter poi dimostrare, in caso di sospetto, la propria estraneità, esibendo una scrittura diversa da quella impressa sul famigerato documento recuperato nel cestino della carta dall'addetta alle pulizie Madame Bastian, impiegata presso l'Ambasciata tedesca a Parigi. Fu Jules Crépiux-Jamin, perito di parte di Dreyfus, insieme ad altri colleghi di fama internazionale, ad accertare dapprima la non appartenenza del documento a Dreyfus e poi a ricondurlo al vero autore, ossia Esterhazy (CRÉPIEUX-JAMIN, Jules. L'expertise en écritures et les leçons de l'affaire Dreyfus. Année psychologique, Paris, v. 13, 1906, pp. 187-229. Si veda anche LOCARD, Edmond. L'affaire Dreyfus et l'expertise des documents écrits. La Giustizia penale, Roma, v. 44, 1938, pp. 159-192).

${ }^{124}$ Art. 166 del c.p.c del 1913: «Gli ufficiali di polizia giudiziaria sequestrano le cose che servirono o furono destinate a commettere il reato, quelle che ne sono il prodotto, e tutto ciò che possa essere utile all'accertamento della verità. Nelle loro operazioni possono procedere, se sia il caso, anche a rilievi tecnici e fotografici». Una certa preoccupazione aveva accompagnato l'elaborazione del codice Finocchiaro Aprile dal momento che nella stesura originaria conteneva limitazioni nelle funzioni di polizia giudiziaria ai funzionari di P.S.: «Siamo pratici, è possibile fare la polizia giudiziaria senza il contributo efficace, pronto della pubblica sicurezza? Potremo avere specialmente col tempo dei magistrati i quali avranno la più completa cultura ma non potranno agire senza il concorso di capaci funzionari di P.S.» (OTTOLENGHI, Salvatore. Il nuovo progetto del Codice di procedura penale e le funzioni della P.S. nella polizia giudiziaria. Bollettino della Scuola di polizia scientifica e del servizio di segnalamento, Roma, fasc. 3, 1912, p. 85, ma si veda in generale pp. 83-92).

${ }^{125}$ Alle spalle del c.p.p del 1913 vi era una circolare del 24 luglio 1910 dell'allora Ministro di Grazia e Giustizia del governo Luzzati, Cesare Fani, favorevole a 
le impronte rilevate sul luogo o sul corpo del reato e quelle di individui indiziati o sospettati ed il relativo giudizio sull'identità non costituivano piú un'esclusiva funzione peritale ma potevano esser svolti dagli ufficiali di polizia giudiziaria a ciò istruiti. La novità, apparentemente tecnica, aveva tuttavia conseguenze importanti, dal momento che consentiva ai sensi degli articoli 162 e del già citato 166 del c.p.p. del 1913 che l'attività in questione fosse fatta «di propria iniziativa dall'ufficiale di polizia giudiziaria», allo scopo di «fornire all'autorità giudiziaria le cognizioni che possono condurre alla identificazione dei colpevoli».

Si trattava anche di una chiara indicazione rivolta ai magistrati: essi dovevano, per primi, conoscere quali fossero e in che cosa consistessero tali indagini scientifiche per coglierne la portata ed essere pertanto in grado di richiedere e utilizzare l'opera di funzionari ben addestrati. Il cambio di passo richiesto alla polizia finiva inevitabilmente per coinvolgere l'autorità giudiziaria, sia nella fase istruttoria che giudicante. Si sarebbe dovuto perseguire un allineamento di competenze, tanto con riguardo ad aspetti meramente formali (limiti e compiti della polizia giudiziaria e di pubblica sicurezza in generale) che di contenuto. Se compito della Scuola era preparare i funzionari, spettava ai magistrati evitare che tale patrimonio di conoscenze rimanesse inutilizzato.

Fu tuttavia l'art. 223 del c.p.p. del 1930 (in ragione anche di una coerente evoluzione teorica ma soprattutto di un'accertata valenza pratica, derivata da continua sperimentazione ${ }^{126}$ ) a prevedere in maniera esplicita che «agli accertamenti, ai rilievi segnaletici, descrittivi o fotografici e ad

una diffusione del tecnicismo scientifico nelle indagini giudiziarie, segnando una riforma metodologica di primaria importanza. La circolare è consultabile in Bollettino della Scuola di polizia scientifica e del servizio di segnalamento. Roma, fasc. 2, 1911, pp. 15-18. Ma si attribuiva a Silvio Longhi, primo insegnante di diritto e procedura penale della Scuola nel 1907, il merito di avere, di fatto, attraverso il contributo alla redazione di tale norma, riconosciuto ufficialmente il lavoro svolto dalla Scuola, avendo sperimentato con mano l'efficacia pratica dei metodi in essa insegnati (FALCO, Giuseppe. Salvatore Ottolenghi cit., p. 6).

${ }^{126}$ La tecnica era così diffusa che si ricorda che dai 26 gabinetti di Polizia scientifica del Regno presenti sul territorio nazionale al momento dell'entrata in vigore del c.p.p. del 1913 si era passati a 62. 
ogni altra operazione tecnica relativa alla loro funzione, gli ufficiali di Polizia Giudiziaria procedono direttamente» ${ }^{127}$.

L'art. 233 non ricorreva più a quella impacciata facoltà concessa dall'abrogato art. 166, ma in modo inequivocabile ammetteva l'importanza delle prove tecnico-scientifiche, attribuendone l'assolvimento alla polizia $^{128}$. Il metodo scientifico introdotto per formare un nuovo corpo polizia aveva alla fine prodotto un'estensione dell'attività inquirente e una compenetrazione, più che interazione, tra magistratura e polizia stessa ${ }^{129}$.

Il cerchio si era chiuso. Il sogno vagheggiato a partire dal 1902 aveva trovato finalmente piena cittadinanza nell'ordinamento. E non può tacersi che ciò fosse avvenuto a vantaggio della polizia d'un regime nient'affatto democratico. Le tecniche della scuola divennero infatti l'alibi per utilizzare a fini politici i dati sulla pericolosità dei singoli individui e controllare (per ridurre al silenzio) possibili avversari e nemici del

${ }^{127}$ Le indagini da compiersi durante un sopralluogo erano regolate dagli artt. 219, 222, 223 del c.p.p del 1930. Al contempo, l'art. 133 del codice penale fascista in qualche modo faceva propri gli insegnamenti della Scuola positiva e di quelli impartiti nella Scuola di polizia scientifica, laddove il giudice, per determinare la pena, doveva tenere conto della capacità a delinquere del colpevole, desunta da criteri che ricalcavano un approccio fisico-antro-sociologico. Le aule dei tribunali si erano dunque, con atto imperativo, aperte all'antropologia e alla psicologia criminale, sul presupposto che il magistrato sapesse studiare un delinquente e diagnosticarne la natura e la pericolosità, esaminandone la vita e il delitto commesso. Tanto entusiastico ardore verso il codice del 1930 forse trova una spiegazione anche in un episodio della vita di Ottolenghi, riportato da Falco: «nell'ottobre del 1922 il camerata Salvatore Ottolenghi, genio rivoluzionario, con giovanile baldanza, marciava indrappellato coi Fascisti della Marcia su Roma e cantava con loro gli inni della giovinezza fascista» (FALCO, Giuseppe. Salvatore Ottolenghi cit., p. 14). Ottolenghi guardò con favore all'ascesa del fascismo, anche se questo «avrebbe mandato in frantumi quell'Italia liberale che egli si era così impegnato a riformare» (LABANCA, Nicola. Salvatore Ottolenghi cit., p. 21).

128 «Queste nuove armi, che la scienza ha offerto alla Polizia, di indiscutibile valore per la difesa della Società dai delinquenti e che la rigenerata legislazione fascista ha accolto e valorizzato, non possono rimanere inoperose in un Regime la cui opera di difesa sociale non ha avuto e non ha l'eguale per efficacia e vastità» (GIRI, Emilio. Il "ritratto parlato” del sopralluogo cit., p. 176).

${ }^{129}$ RONDINI, Paolo. Polizia giudiziaria e magistratura nell'Italia liberale. Historia et ius, n. 11, giugno 2017, paper n. 16, pp. 1-42 (http://www.histroiaetius.eu). 
regime ${ }^{130}$, finendo per tradire lo spirito con cui la scuola era nata. Si può sostenere in fondo che Ottolenghi operò in equilibrio funambolico tra spinte scientifiche eterogenee e climi politici contraddittori, attraversando la transizione dall'Italia liberale a quella fascista, alla perpetua ricerca di sostenitori della creatura di cui andava più fiero: la sua Scuola ${ }^{131}$.

\section{Considerazioni conclusive}

La creazione della polizia scientifica in Italia rappresenta un frammento, sottovalutato per un certo tempo dalla storiografia e solo recentemente divenuto oggetto di specifici studi, ma tutt'altro che marginale nella prassi giudiziaria, di quella vera e propria invasione di studi, tecniche e metodologie che, nell'età del positivismo imperante, dalle scienze naturali trasmigrò nel penale tra entusiasmi e resistenze. Siamo agli albori, come ognuno sa, della criminologia, della psicologia, delle scienze sociali che promettevano, o minacciavano, di trasformare il giudice in un clinico e la polizia, fosse di pubblica sicurezza o giudiziaria, in un organo specializzato che sempre piú avrebbe dovuto affinare competenze tecniche e sperimentali ${ }^{132}$. Affonda qui le sue radici la cd. prova scientifica, dal cui contributo ci si attendevano passi decisivi verso la certezza processuale o, almeno, la drastica riduzione dei margini d'errore.

L'irruzione della scienza nel processo finiva per ridimensionarne la dimensione integralmente giuridica. Non era nuova, d'altronde, l'ambizione di incanalare l'arbitrio del giudice (poi 'edulcorato' come libero convincimento) entro perimetri prestabiliti. Come, ai tempi dell'aristotelismo scolastico, la prova legale aveva assicurato un meccanismo calcolabile e controllabile di ricerca del verum, cosí tra Otto e Novecento

${ }^{130}$ GIBSON, Mary. Nati per il crimine cit., passim; LABANCA, Nicola. Complessità della storia di polizia (compresa quella scientifica). In: Una cultura pofessionale cit., pp. 13-22.

${ }^{131}$ Sul punto si veda la riflessione di LABANCA, Nicola. Salvatore Ottolenghi cit., pp. 28-32.

${ }^{132}$ MILETTI, Marco. Ritorno all'inquisizione. Scuola positiva e pulsioni autoritarie nel processo penale italiano. Diritto penale XXI secolo, Padova, n. 10, v. 2, 2011, pp. 455-492. 
si sperava che la scienza, grazie alla sua oggettività, avrebbe condotto anche la giustizia ad approdi certi e incontrovertibili.

La storia e l'esperienza hanno ridimensionato simili atti di fede. L'epistemologia del Novecento ha messo in seria discussione ogni pretesa di 'oggettività' assoluta dei meccanismi cognitivi. Nella storia del processo penale il sogno d'un giudice bocca della scienza è tramontato come era accaduto al montesquieviano giudice bocca della legge. La gran parte del contenzioso giudiziario resta incardinato su elementi indiziari, che implicano la libera valutazione del giudice: in mancanza di prove 'piene' tradizionali (confessioni o testimonianze), i riscontri scientifici (la disposizione delle gocce di sangue, la posizione del cadavere, la descrizione degli oggetti rinvenuti durante il sopralluogo, l'indagine psicologica del sospettato, via discorrendo) richiedono una ricomposizione sintetica che non può che affidarsi alla prudentia dell'interprete, quasi a riproporre l'antico adagio quae singula non prosunt collecta iuvant ${ }^{133}$.

Vi è forse un uso e un abuso del termine 'scientifico' nell'ambito del processo penale, sull'onda entusiastica d'un progresso che, in certe fasi come quella qui esaminata, ha indotto ad attribuire al risultato investigativo una patente di verità assoluta, oltre che una deresponsabilizzazione delle singole scelte e decisioni. L'insistenza con cui, al tempo di Ottolenghi e dei suoi allievi, si richiedeva un'adeguata preparazione ai funzionari di polizia e ai magistrati dimostrava un cambiamento di prospettiva nell'acquisizione e nella valutazione delle prove. Il iudex peritus peritorum non era più il magistrato onnisciente pensato in età medievale, bensí un funzionario di Stato di estrazione pur sempre giuridica, ma sufficientemente attrezzato per esercitare un controllo sulle prove scientifiche; per valutare, respingere, criticare le perizie tecniche; per non restare recettore passivo del verbo degli scienziati ma proporsi credibilmente come decisore ultimo della regolarità formale e sostanziale di tutte le acquisizioni probatorie.

Da un'idea di scientizzazione del processo, che sostituiva nuove prove e ne accontonava altre apparentemente usurate dal tempo (prima

${ }^{133}$ Se infatti i dati offerte dalla "scienze ausiliarie" «non possono offrire prove dirette, valgono però come indizii efficaci, che congiunti ad altri, possono mettere la giustizia penale sulla via del vero» [RIGHINI, (studente in legge). I procedimenti indiziari e l'antropologia criminale. Archivio di psichiatria, scienze penali ed antropologia criminale, Torino, v. 2, 1881, p. 169]. 
tra tutte, la testimonianza), la seconda metà del Novecento si è mossa in direzione d'una processualizzazione del metodo scientifico. I timori circa l'invasività degli strumenti di indagine tecnica si sono intensificati man mano che si è rafforzata l'esigenza di tutela dei diritti fondamentali e della sfera individuale. Il dibattito non si è sopito ed anzi ha trovato in anni recenti nuova linfa tra i processualpenalisti con riguardo al rapporto tra perito e consulente e tra consulente dell'accusa e della difesa ${ }^{134}$. Si avverte la preoccupazione di esorcizzare i residui dell'impianto autoritario costruito dal codice Rocco ${ }^{135}$ e di affrancarsi da quell'inconscio inquisitorio ${ }^{136}$ tuttora incombente sul rito penale italiano.

\section{Biblografia}

ALGERI, Giovanni. Alcuni casi di ipnotismo di criminali pazzi. Archivio di psichiatria, scienze penali e antropologia criminale, Torino e Roma, v. 8, 1887.

ALONGI, Giuseppe. Polizia e delinquenza in Italia. Roma: Soriano nel Cimino, 1887.

ANFOSSO, Luigi. Il casellario giudiziale centrale. Studio sulla identificazione dei delinquenti e designazione dei loro contrassegni personali. Torino: Fratelli Bocca, 1895.

ANFOSSO, Luigi. Il segnalamento dei delinquenti e il nuovo antropometro popolare. Archivio di Psichiatria, Scienze penale ed antropologia criminale, Torino, n. 4, v. 9, 1888.

${ }^{134}$ Per tutti cfr. CANZIO, Giovanni e LUPÀRIA, Luca. Prova scientifica e processo penale. Padova, 2018; KOSTORIS, Roberto. Una grave mistificazione inquisitoria: la pretesa fede privilegiata del responso del consulente tecnico dell'accusa. Sistema penale, 28 settembre 2020 (https://www.sistemapenale.it/it/scheda/kostoris-cassazione-16458-2020-fede-privilegiata-consulente-tecnico-pm); CONTI, Carlotta. La prova scientifica alle soglie dei vent'anni dalla sentenza Franzese: vette e vertgini in epoca di pandemia. Sistema penale, 9 febbraio 2021 (https://www.sistemapenale.it/it/articolo/ conti-prova-scientifica-venti-anni-sentenza-franzese).

135 GARLATI, Loredana. Contro il sentimentalismo. L'impianto inquisitorio del sistema delle prove nel c.p.p. del 1930. Criminalia, Pisa, 2012, pp. 181-227

${ }^{136} \mathrm{Mi}$ permetto di riprendere il titolo di un volume da me curato, L'inconscio inquisitorio. L'eredità del Codice Rocco nella processualpenalistica italiana. Milano, 2010. 
ANFOSSO, Luigi. La lotta contro i delinquenti. Conferenza tenuta nella società filotecnica di Torino 27 maggio 1892. Borgo San Dalmazzo, 1892.

BECCARIA, Cesare. Dei delitti e delle pene. Edizione Nazionale delle Opere di Cesare Beccaria, I, Milano, 1984.

BERENINI, Agostino. Azione ed istruzione penale. Organi e funzioni. Parma, 1888.

BERTILLON, Alphonse. Sur le fonctionnement du service des signalements anthropométriques. Archives de l'anthropométrie criminel, Paris, t. 3, 1888.

BERTILLON, Alphonse. La photographie judiciaire avec un appendice sur la classification et l'identification anthropométriques. Paris, 1890.

BERTILLON, Alphonse. Identification anthropométrique Instructions signalétiques. Melun, 1893.

BERTILLON, Alphonse. Notice sur le fonctionnement du Service d'Identification de la Préfecture de Police de Paris. Paris, 1889.

BERTILLON, Alphonse. Resultats obtenus par l'antropometrie au point de vue de la criminalité. Rapport au Congres international de Geneve. Archives de l'anthropologie criminelle. Paris, t. 11, 1896.

BERTILLON, Suzanne. Vie d'Alphonse Bertillon. Paris, 1941.

BISCEGLIA, Roberto. La Scuola di Polizia Scientifica. Noi e il mondo. Rivista mensile de La Tribuna, anno 13, n. 1, 1923.

BONOMI, Giuseppe. Project of an Instrument for the Identification of Persons for use in military establishments, police offices etc.. London, 1872.

BRUNELLI, David. Recidiva e Scuola positiva nella disciplina del Codice Rocco. Spunti di riflessione. Diritto penale XXI secolo, Padova, n. 2, 2011.

BRUNELLO, Piero. Storie di anarchici e di spie. Polizia e politica nell'Italia liberale. Roma, 2009.

BRUSA, Emilio. La morale e il diritto criminale al limbo. Discorso inaugurale letto nella Regia Università di Torino l'8 marzo 1880. Torino, 1880.

BRUSA. Emilio, Legislazione, dottrina e giurisprudenza penale italiana negli anni 1890-91. Annuario di dottrina, legislazione e di giurisprudenza, Milano, v. 3, 1891

BUZZANCA, Salvatore. La figura di Salvatore Ottolenghi. In: http://ssaistorico. interno.gov.it/download/allegati1/instrumenta_16_14_buzzanca.pdf 
CAMPOSANO, Raffaele. La polizia scientifica in Italia. In: LABANCA, Nicola e DI GIORGIO, Michele (a cura di). Salvatore Ottolenghi. Una cultura professionale per la polizia dell'Italia liberale e fascista. Antologia degli scritti (1883-1934). Milano, 2018.

CANZIO, Giovanni e LUPÀRIA, Luca. Prova scientifica e processo penale. Padova, 2018.

CARFORA, Francesco. Polizia giudiziaria. In: Digesto Italiano. Torino, v. XVIII, parte seconda, 1906-1912.

COMPAGNONE, Luigi. Il casellario giudiziario ed il metodo Bertillon per accertare l'identità dei recidivi: studio e proposte. Napoli 1888.

CONTI, Carlotta. La prova scientifica alle soglie dei vent'anni dalla sentenza Franzese: vette e vertgini in epoca di pandemia. Sistema penale, 9 febbraio 2021 (https://www. sistemapenale.it/it/articolo/conti-prova-scientifica-venti-anni-sentenza-franzese).

COSENZA, Vincenzo. Il casellario giudiziale. Roma, 1895.

COSENZA, Vincenzo. Relazione sul casellario presentata alla Commissione per la statistica giudiziaria nella sessione del maggio 1895. Giurisprudenza italiana, Torino, v. 47, parte quarta, 1895.

CRÉPIEUX-JAMIN, Jules, L'expertise en écritures et les leçons de l'affaire Dreyfus. Année psychologique, Paris, v. 13, 1906.

D’AMELIO, Mariano. I progressi della polizia scientifica. Bollettino della Scuola superiore di polizia e dei servizi tecnici annessi, Roma, fasc. 21, 1933.

D'ANTONIO, Emanuele. Lombroso, Ottolenghi e le origini della polizia scientifica italiana. In: LABANCA, Nicola e DI GIORGIO, Michele (a cura di). Una cultura professionale per la polizia dell'Italia liberale e fascista. Antologia del "Bollettino della Scuola di polizia scientifica (1910-1939)". Milano, 2020.

DARMON, Pierre. Bertillon, le fondateur de la police scientifique. L'Histoire, n. 105, novembre 1987.

DE BLASIO, Abele. L'orecchio dei Napoletani normali e criminali. Archivio di psichiatria, neuropatologia, antropologia criminale e medicina legale, Milano-Torino, v. 26 (v. 2 della Serie 3), 1905.

DE NOTARISTEFANI, Raffaele. L'identificazione del delinquente (bertillonage). Napoli, 1898.

DI GIORGIO, Michele. Dalla medicina all'investigazione. Il contributo di Salvatore Ottolenghi per la costruzione di un sapere scientifico di polizia. In: LABANCA, 
Nicola e DI GIORGIO, Michele (a cura di). Salvatore Ottolenghi. Una cultura professionale per la polizia dell'Italia liberale e fascista. Antologia degli scritti (1883-1934). Milano, 2018.

DI GIORGIO, Michele. Un laboratorio per la costruzione e la diffusione dei saperi. La scuola di polizia scientifica e il "bollettino" (1910-1939). In: LABANCA, Nicola e DI GIORGIO, Michele (a cura di). Una cultura professionale per la polizia dell'Italia liberale e fascista. Antologia del "Bollettino della Scuola di polizia scientifica (1910-1939)”. Milano, 2020.

DI TULLIO, Benigno. La profilassi criminale nella polizia. Bollettino della Scuola superiore di Polizia e dei servizi tecnici annessi, Roma, fasc. 14-16, 1937.

ELLERO, Umberto. La fotografia nelle funzioni di polizia e processuali. Milano, 1908.

FALCO, Giuseppe, Polizia scientifica e giurisprudenza. Bollettino della Scuola di polizia scientifica e del servizio di segnalamento, Roma, fasc. 7-8, 1921.

FALCO, Giuseppe. "Identità”. Metodo scientifico di segnalamento e di identificazione. Roma, 1922.

FALCO, Giuseppe. Evoluzione della "cartella biografica” e pericolosità. Bollettino della Scuola superiore di polizia e del servizio di segnalamento, Roma, fasc. 14-15, 1926.

FALCO, Giuseppe. Salvatore Ottolenghi. Bollettino della Scuola superiore di polizia e dei servizi tecnici annessi, Roma, fasc. 22-23, 1935, pp. 3-16.

FERRI, Enrico. Diritto penale ed antropologia criminale (Studi critici sull'Uomo delinquente). Archivio di psichiatria, antropologia criminale e scienze penali, Torino e Roma, v. 1, 1880.

FERRI, Enrico. Sociologia criminale. Napoli, 1892.

FRANCHI, Bruno, Il principio individualizzatore nell'istruttoria penale. La Scuola Positiva, Milano, n. 11, v. 10, 1900.

FRIGERIO, Luigi. L'oreille externe. Étude d'anthropologie criminelle. Parys et Lion, 1888. Pubblicato anche in Archives de l'anthropologie criminelle, Paris, v. 3, 1888.

GALTON, Francis. Finger prints. London, 1892.

GARLATI, Loredana (a cura di). L'inconscio inquisitorio. L'eredità del Codice Rocco nella processualpenalistica italiana. Milano, 2010.

GARLATI, Loredana. Contro il sentimentalismo. L'impianto inquisitorio del sistema delle prove nel c.p.p. del 1930. Criminalia, Pisa, 2012. 
GAROFALO, Raffale. Il codice per i galantuomini. Roma, 1902.

GASTI, Giovanni. Il corso di Polizia scientifica. Roma, 1903.

GASTI, Giovanni. L'identificazione dei delinquenti e la funzione di polizia nell'attuale momento giuridico e sociale. Prolusione al corso di Segnalamento detta alla Scuola di Polizia Scientifica il 2 maggio 1910. Bollettino della Scuola di polizia scientifica e del servizio di segnalamento, Roma, fasc. 1, 1910.

GASTI, Giovanni. L'identificazione dei delinquenti e la funzione di polizia nell'attuale momento giuridico e sociale. Prolusione al corso di Segnalamento detta alla Scuola di Polizia Scientifica il 2 maggio 1910 (continuazione). Bollettino della Scuola di polizia scientifica e del servizio di segnalamento, Roma, fasc. 2, 1911.

GASTI, Giovanni. La missione attuale dell'antrometria nelle funzioni di polizia. Bollettino della Scuola di polizia scientifica e del servizio di segnalamento, Roma, fasc. 3, 1912.

GIANNELLI, Augusto; PARDO, Giorgio. I sistemi di segnalazione antropometrica. Osservazioni e proposte. Rivista di discipline carcerarie, parte prima, Roma, anno 23, n. 10, 1898.

GIBSON, Mary. Nati per il crimine. Cesare Lombroso e le origini della criminologia biologica. Milano, 2002.

GILARDI, Ando. Wanted! Storia, tecnica ed estetica della fotografia criminale, segnaletica e giudiziaria. Milano, 2003.

GIRI, Emilio. "Il ritratto parlato del sopraluogo". La Giustizia penale (parte prima: I presupposti del diritto e della procedura penale), Roma, v. 40 (10 della $4^{\text {a }}$ serie), 1934.

GIRI, Emilio. Evoluzione del ritratto parlato “Ottolenghi” del sopralluogo. Bollettino della Scuola di polizia scientifica e dei servizi tecnici annessi, Roma, fasc. 12-13, 1925.

GIRI, Emilio. Il "ritratto parlato" del sopralluogo e l'art. 233 del codice di procedura penale. Bollettino della Scuola superiore di polizia e dei servizi tecnici annessi, Roma, fasc. 21, 1933.

GIRI, Emilio. Il "ritratto parlato" del sopralluogo nella pratica. Bollettino della Scuola superiore di polizia e dei servizi tecnici annessi, Roma, fasc. 21, 1933.

GIRI, Emilio. L'applicazione del metodo segnaletico descrittivo e le comuni prove sperimentali nella indagine giudiziaria. Bollettino della Scuola superiore di polizia e dei servizi tecnici annessi, Roma, fasc. 22-23, 1935.

GIULIANO, Andrea. Salvatore Ottolenghi. Le impronte digitali in polizia scientifica e medicina legale. Torino, 2018. 
GRADENIGO, Giuseppe. L'orecchio nei delinquenti. Giornale della Regia Accademia di medicina di Torino, Torino, v. 37, 1889.

GRADENIGO, Giuseppe. La conformazione del padiglione dell'orecchio nei normali, negli alienati e nei delinquenti. Giornale della Regia Accademia di medicina di Torino, Torino, v. 38, 1890.

KOSTORIS, Roberto. Una grave mistificazione inquisitoria: la pretesa fede privilegiata del responso del consulente tecnico dell'accusa. Sistema penale, 28 settembre 2020 (https://www.sistemapenale.it/it/scheda/ kostoris-cassazione-16458-2020-fede-privilegiata-consulente-tecnico-pm).

LABANCA, Nicola. Salvatore Ottolenghi e i saperi di polizia. Per una biografia. In: LABANCA, Nicola e DI GIORGIO, Michele (a cura di). Ottolenghi Salvatore. Una cultura professionale per la polizia dell'Italia liberale e fascista. Antologia degli scritti (1883-1934). Milano, 2018.

LABANCA, Nicola. Complessità della storia di polizia (compresa quella scientifica). In: LABANCA, Nicola e DI GIORGIO, Michele (a cura di). Una cultura professionale per la polizia dell'Italia liberale e fascista. Antologia del "Bollettino della Scuola di polizia scientifica (1910-1939)”. Milano, 2020.

LACASSAGNE, Alexandre. Alphonse Bertillon. L'homme. Le savant. La pensée philosophique. Archives de l'Anthropologie Criminelle, Lyon, t. 29, 1914.

LOCARD, Edmond. L'affaire Dreyfus et l'expertise des documents écrits. La Giustizia penale, Roma, v. 44, 1938.

LOCARD, Edmond. La preuve judiciaire par les empreintes digitales. Archives de l'anthropologie criminelle, Paris, t. 29, 1914.

LOCATELLI, Paolo. Sorveglianti e sorvegliati. Appunti di fisiologia sociali presi dal vero. Milano, 1876.

LOMBROSO, Cesare. Discours d'ouverture au VI Congrès d'Anthropologie criminelle. Archives d'Anthropologie criminelle, de Criminolgie et de Psychologie normale et pathologique, Lyon-Paris, t. 21, 1906.

LOMBROSO, Cesare. Innocenza di gravissima imputazione dimostrata dalla Antropologia criminale. Archivio di psichiatria, scienze penali e antropologia criminale, Torino e Roma, v. 23 (v. 7 della serie 2), 1902.

LOMBROSO, Cesare. L'ipnotismo applicato alla procedura penale. Archivio di psichiatria, scienze penali e antropologia criminale, Torino, v. 8, 1887.

LOMBROSO, Cesare. Studi sull'ipnotismo. Roma Torino Firenze, $3^{\text {a }}$ ed., 1887. 
LOMBROSO, Cesare. Sull'incremento del delitto in Italia e sui mezzi per arrestarlo. Torino, 1879.

LOMBROSO, Cesare. L'uomo delinquente in rapporto all'antropologia, alla giurisprudenza ed alla psichiatria. Torino, quinta edizione, v. 3, 1897.

LOMBROSO, Cesare; OTTOLENGHI, Salvatore. Nuovi studi sull'ipnotismo e sulla credulità. Archivio di psichiatria, scienze penali e antropologia criminale, Torino, n. 1 , v. 11,1890 .

LOMBROSO Cesare; BIANCHI, Augusto Guido. Il caso Olivo. Milano, 1905.

MARTINELLI, Giovani. Cenni storici su l'Università di Ferrara. Ferrara, 1908.

MARTINI, Paolo; PIVA, Italo. Salvatore Ottolenghi e la criminologia positivista. In: Per una storia dell'insegnamento della medicina legale nell'Università di Siena. Siena, 1993.

MASINI, Eliseo. Sacro arsenale overo Prattica dell'officio della Santa Inquisitione, Seconda Parte, Del modo di formare i processi, e essaminare Testimoni, e Rei. Bologna, 1665.

MELIS, Guido. La macchina imperfetta. Immagine e realtà dello Stato fascista. Bologna, 2018.

MILETTI, Marco Nicola. La follia nel processo. Alienisti e procedura penale nell'Italia postunitaria. Acta Histriae, Koper, n. 15, vol. 1, 2007.

MILETTI, Marco. Ritorno all'inquisizione. Scuola positiva e pulsioni autoritarie nel processo penale italiano. Diritto penale XXI secolo, Padova, n. 10, vol. 2, 2011.

MORSELLI, Enrico. Critica e riforma del metodo in antropologia fondate sulle leggi statistiche e biologiche dei valori seriali. Roma, 1880.

MUZZARELLI, Federica. Formato tessera. Storia, arte, e idee in photomatic. Milano, 2003.

OLIVIERI, Vittorio. Un delinquente per passione. La Scuola positiva, Milano, n. $2,1895$.

OTTOLENGHI Salvatore. Polizia empirica e polizia scientifica. Palermo, 1897.

OTTOLENGHI, Salvatore. Il nuovo progetto del Codice di procedura penale e le funzioni della P.S. nella polizia giudiziaria. Bollettino della Scuola di polizia scientifica e del servizio di segnalamento, Roma, fasc. 3, 1912. 
OTTOLENGHI, Salvatore. Ai lettori. Bollettino della Scuola di polizia scientifica e del servizio di segnalamento, Roma, fasc. 1, 1910.

OTTOLENGHI, Salvatore. Alfonso Bertillon. Bollettino della Scuola di polizia scientifica e del servizio di segnalamento, Roma, fasc. 4, 1914.

OTTOLENGHI, Salvatore. Diffusione e risultati del Servizio d'investigazione giudiziaria nel quadriennio 1915-1918. Bollettino della Scuola di polizia scientifica e del servizio di segnalamento, Roma, fasc. 7-8, 1921.

OTTOLENGHI, Salvatore. Il segnalamento del delinquente in servizio della polizia giudiziaria. Palermo, 1898.

OTTOLENGHI, Salvatore. Il segnalamento descrittivo e le funzioni della polizia. Bollettino della Scuola di polizia scientifica e dei servizi tecnici annessi, Roma, fasc. 3, 1912 .

OTTOLENGHI, Salvatore. Insegnamento di investigazioni giudiziarie. Bollettino della Scuola di polizia scientifica e del servizio di segnalamento, Roma, fasc. 1, 1910.

OTTOLENGHI, Salvatore. Introduzione alle indagini tecniche di polizia giudiziaria. Bollettino della Scuola di polizia scientifica e dei servizi tecnici annessi, Roma, fasc. $21,1933$.

OTTOLENGHI, Salvatore. L'insegnamento universitario della polizia giudiziaria scientifica. Torino, 1897.

OTTOLENGHI, Salvatore. La nuova “cartella biografica dei pregiudicati”. Bollettino della Scuola di polizia scientifica e del servizio di segnalamento, Roma fasc. 5-6, 1916.

OTTOLENGHI, Salvatore. La perizia di scrittura e la identificazione grafica. Bollettino della Scuola di polizia scientifica e dei servizi tecnici annessi, Roma, fasc. 9-11, 1923.

OTTOLENGHI, Salvatore. La personalità del delinquente e la "cartella italiana biografica del pregiudicato" alla IV riunione della commissione internazionale di polizia criminale (6-8 luglio 1927) ad Amsterdam. Bollettino della Scuola superiore di polizia e del servizio di segnalamento, Roma, fasc. 16-17, 1928.

OTTOLENGHI, Salvatore. La Scuola di Polizia scientifica e il servizio di segnalamento in Italia 1902-1910. Cenni storici. Bollettino della Scuola di polizia scientifica e del servizio di segnalamento. Roma, fasc. 1, 1910.

OTTOLENGHI, Salvatore. La suggestione e le facoltà psichiche occulte in rapporto alla pratica legale e medico-forense. Torino, 1900. 
OTTOLENGHI, Salvatore. Polizia scientifica biologia e antropologia criminale. Bollettino della Scuola di polizia scientifica e del servizio di segnalamento, Roma, fasc. 3, 1912.

OTTOLENGHI, Salvatore. Prefazione a FALCO Giuseppe. "Identità". Metodo scientifico di segnalamento e di identificazione. Roma, 1922.

OTTOLENGHI, Salvatore. Relazione degli anni 1919, 1920 e 1921. Bollettino della Scuola di polizia scientifica e dei servizi tecnici annessi, Roma, fasc. 9-11, 1923.

OTTOLENGHI, Salvatore. Trattato di polizia scientifica. Milano, v. I, 1910.

OTTOLENGHI, Salvatore. Trattato di polizia scientifica. Milano, v. II, 1932.

OTTOLENGHI, Salvatore. Una "polizia scientifica” antiscientifica. La Scuola Positiva, Milano, v. 1 dell'anno 21, 1911.

OTTTOLENGHI, Salvatore. Il colore e la forma delle cicatrici antiche in rapporto alla medicina forense (comunicazione fatta alla R. Accademia di Medicina di Torino nella seduta del 20 dicembre 1889). Giornale della R. Accademia di Medicina di Torino, Torino, v. 37, 1889.

OTTOLENGHI, Salvatore. Seconda conferenza internazionale di Polizia - New York 12-16 maggio 1925. Bollettino della Scuola superiore di polizia e dei servizi tecnici annessi, Roma, fasc. 14-15, 1926.

OTTOLENGHI, Salvatore. L'opera di Cesare Lombroso e la polizia scientifica. In: L'opera di Cesare Lombroso nella scienza e nelle sue applicazioni. Milano-Torino-Roma, 1908.

PACINOTTI, Giovanni. Una Scuola di polizia scientifica nell'Università di Ferrara. Rivista di diritto penale e sociologia criminale, Pisa, anno V, 1904.

PESSINA, Enrico. Il naturalismo e le scienze giuridiche. Discorso inaugurale letto nella Regia Università di Napoli il 17 dicembre 1878. Napoli, 1879.

PIFFERI, Michele. L'individualizzazione della pena. Difesa sociale e crisi della legalità penale tra Otto e Novecento. Milano, 2013.

PUGLIA, Ferdinando. Prolegomeni allo studio del diritto repressivo. Roma Torino Firenze, 1883.

QUETELET, Adolphe. Phisique sociale ou essai sur le développement des facultés de l'homme. Bruxelles, Paris, Saint-Pétersbourg, t. I, 1869.

QUETELET, Adolphe. Sur la statistique morale et les principes qui doivent en former la base. Mémoires de l'Académie Royale des Sciences, des Lettres et des Beaux-Arts de Belgique, Bruxelles, v. 21, 1848. 
RIGHINI, (studente in legge). I procedimenti indiziari e l'antropologia criminale. Archivio di psichiatria, scienze penali ed antropologia criminale, Torino, v. 2, Torino, 1881.

RONDINI, Paolo. Polizia giudiziaria e magistratura nell'Italia liberale. Historia et ius, n. 11, giugno 2017, paper n. 16, pp. 1-42 (http://www.histroiaetius.eu).

RUISTIUCCI, Luigi. Nel regno dei ladri. Come si ruba e come si falsificano le monete. Bologna, 1919.

ROSSI, Virgilio. Polizia Empirica e Polizia Scientifica (Idee vecchie e fatti nuovi). Aquila, 1898.

ROSSI, Virgilio. Polizia empirica e polizia scientifica. Il Tachiantropometro Anfosso applicato ad una centuria di criminali. Rivista di discipline carcerarie, Roma, anno 19, 1889.

SBRICCOLI, Mario. La penalistica civile. Teorie e ideologie del diritto penale nell'Italia unita. In: SBRICCOLI, Mario. Storia del diritto penale e della giustizia. Scritti editi e inediti (1972-2007). Milano, t. I, 2009.

SBRICCOLI, Mario. Polizia. In: SBRICCOLI, Mario. Storia del diritto penale e della giustizia. Scritti editi e inediti (1972-2007). Milano, t. I, 2009.

SCHETTINI, Laura. Ottolenghi Salvatore. In: Dizionario Biografico degli Italiani. Roma, v. 79, 2013.

SCHETTINI, Laura. Per lo studio della personalità del delinquente. La polizia scientifica e il servizio di identità. In: LABANCA, Nicola e DI GIORGIO, Michele (a cura di). Una cultura professionale per la polizia dell'Italia liberale e fascista. Antologia del "Bollettino della Scuola di polizia scientifica (1910-1939)". Milano, 2020.

SORRENTINO, Ugo. La prova dattiloscopica dinanzi ai magistrati italiani. Bollettino della Scuola superiore di polizia e dei servizi tecnici annessi, Roma, fasc. 22-23, 1935.

TOSATTI, Giovanna. Pericolosi per la sicurezza dello Stato: le schedature della polizia tra periferia e centro. Percorsi storici, n. 0, 2011.

TOSATTI, Giovanna. La repressione del dissenso politico tra l'età liberale e il fascismo. L'organizzazione della polizia. Studi Storici, Roma, n. 38, v. 1, 1997.

TOSATTI, Giovanna. Dal «segnalamento empirico» alla polizia scientifica. In: ANTONIELLI, Livio e LEVATI, Stefano (a cura di). Tra polizie e controllo del territorio: alla ricerca delle discontinuità. Soveria Mannelli, 2017. 
TURATI, Filippo. Il delitto e la questione sociale. Appunti sulla questione penale. Milano, 1883.

VUCETICH, Juan. Dactiloscopia comparada. El nuevo sistema argentino. La Plata, 1904.

ZERBOGLIO, Adolfo. Il rinnovamento scientifico della procedura penale. La Scuola positiva, Milano, anno 4, nn. 18-19, 1894, pp. 817-.

\section{Additional information and author's declarations (scientific integrity)}

Conflict of interest declaration: the author confirms that there are no conflicts of interest in conducting this research and writing this article.

Declaration of authorship: all and only researchers who comply the authorship requirements of this article are listed as authors; all coauthors are fully responsible for this work in its entirety.

Declaration of originality: the author assures that the text here published has not been previously published in any other resource and that future republication will only take place with the express indication of the reference of this original publication; she also attests that there is no third party plagiarism or self-plagiarism. 
Editorial process dates

(http://www.ibraspp.com.br/revista/index.php/RBDPP/about/editorialPolicies)

- Submission: 15/05/2021

- Desk review and plagiarism check: 21/05/2021

- Review 1: 07/06/2021

- Review 2: 09/06/2021

- Review 3: 21/06/2021

- Preliminary editorial decision: 23/06/2021

- Correction round return: 08/07/2021

- Final editorial decision: 16/07/2021

\section{Editorial team}

- Editor-in-chief: 1 (VGV)

- Associated-editor: 1 (RS e GM)

- Reviewers: 3

\section{HOW TO CITE (ABNT BRAZIL):}

GARLATI, Loredana. Alle origini della prova scientifica: la scuola di polizia di Salvatore Ottolenghi. Revista Brasileira de Direito Processual Penal, vol. 7, n. 2, p. 883-934, mai./ago. 2021.

https://doi.org/10.22197/rbdpp.v7i2.597

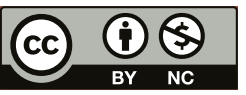

Esta obra está licenciada com uma Licença Creative Commons Atribuição-NãoComercial 4.0 Internacional. 